\title{
Representation of Eye Movements and Stimulus Motion in Topographically Organized Areas of Human Posterior Parietal Cortex
}

\author{
Christina S. Konen ${ }^{1,2}$ and Sabine Kastner ${ }^{1,2,3}$ \\ ${ }^{1}$ Department of Psychology, ${ }^{2}$ Center for the Study of Brain, Mind, and Behavior, and ${ }^{3}$ Princeton Neuroscience Institute, Princeton University, Princeton, \\ New Jersey 08540
}

\begin{abstract}
Recent imaging studies have shown that the human posterior parietal cortex (PPC) contains four topographically organized areas along the intraparietal sulcus (IPS1-IPS4). Using a memory-guided saccade paradigm, we confirmed the locations and retinotopic organization of IPS1-IPS4 and identified two additional areas, IPS5 and superior parietal lobule 1 (SPL1). IPS5 is located at the intersection of the intraparietal and postcentral sulcus; SPL1 branches off the IPS and extends into the superior parietal lobule. Both areas, as well as IPS1-IPS4, each contain a representation of the contralateral visual hemifield. We then probed core functions of the dorsal pathway in these areas, that is, the representation of eye movements and visual motion, to compare the functional characteristics of human PPC to physiologically and anatomically defined areas in monkey PPC. First, as in monkey PPC, a gradient representation of eye movements was found along the IPS with decreasing responses for saccades and increasing responses for smooth pursuit eye movements from posterior/ medial to anterior/lateral. The greatest preference for saccades was found in SPL1 and for smooth pursuit in IPS5. Second, and again similar to monkey PPC, all topographically organized PPC areas responded to different types of motion including planar, circular, and radial optic flow, as assessed using adaptation paradigms. Areas in posterior IPS preferred radial optic flow over planar motion, whereas areas in anterior PPC did not show preference for a particular motion type. Together, our results indicate strikingly similar characteristics in the general functional organization of human and monkey PPC, but also reveal some notable differences.
\end{abstract}

Key words: posterior parietal cortex; intraparietal sulcus; eye movements; visual motion processing; frontal eye fields; fMRI

\section{Introduction}

Most of our current knowledge about the organization of the posterior parietal cortex (PPC) has been derived from invasive studies in nonhuman primates. The intraparietal sulcus (IPS) contains several regions that can be distinguished on the basis of structural and functional criteria and exhibit a variety of different response properties related to the encoding of reaching, grasping, and eye movements, and the processing of multimodal motion, as well as to cognitive operations such as visual attention (Andersen, 1997; Colby and Goldberg, 1999). All regions appear to be associated with the transformation of sensory information into motor output (Ungerleider and Mishkin, 1982; Goodale and Milner, 1992). In contrast to nonhuman primates, less is known about human PPC, partially because of difficulties in distinguishing subdivisions along the IPS using neuroimaging techniques.

Sereno et al. (2001) were the first to find a topographically organized area in human PPC by using a memory-guided saccade task. Recently, four areas located along the IPS and termed IPS1-

Received Jan. 16, 2008; revised June 12, 2008; accepted June 27, 2008.

This work was supported by National Institutes of Health Grants 2R01 MH64043, 1R01 EY017699, and 2P50 MH-62196 (S.K.), and a grant from the German Academic Exchange Service (C.S.K.).

Correspondence should be addressed to Christina S. Konen, Department of Psychology, Center for the Study of Brain, Mind, and Behavior, Princeton University, Green Hall, Princeton, NJ 08540. E-mail:ckonen@princeton.edu. DOI:10.1523/JNEUROSCI.1930-08.2008

Copyright $\odot 2008$ Society for Neuroscience $\quad$ 0270-6474/08/288361-15\$15.00/0
IPS4, have been identified using saccade, spatial attention, and simple fixation tasks. Each IPS area contains a representation of the contralateral visual field and is separated by reversals in the visual field orientation (Sereno et al., 2001; Schluppeck et al., 2005; Sereno and Huang, 2006; Konen and Kastner, 2008). The subdivision of the IPS into distinct areas has permitted the systematic investigation of their functional characteristics. For example, it has been shown that IPS1 is similarly activated by saccadic eye and reaching movements, whereas IPS2 responds preferentially to reaches (Schluppeck et al., 2006; Levy et al., 2007). Furthermore, IPS1 and IPS2 exhibit object-selective responses independent of changes in viewpoint and size, whereas object-selective responses were not found in IPS3 and IPS4 (Konen and Kastner, 2008). Anterior to IPS3-IPS4, the putative human equivalent to the ventral intraparietal area (VIP) in nonhuman primates was defined on the basis of topographically organized, coaligned representations of visual and tactile space and termed the "parietal face area" (Sereno and Huang, 2006). Together, these studies have begun to reveal the functional specialization of human PPC with respect to underlying topographic units. Such approach is particularly useful to probe functional homologies in the organization of human and macaque PPC.

Here, we probed core functions of the dorsal visual pathway, that is, responses evoked by eye movements and visual motion in topographically organized areas of human PPC. Physiology stud- 
ies in monkeys have shown that saccades and smooth pursuit eye movements (SPEMs) are subserved by relatively distinct parietal structures. Neurons in the lateral intraparietal area (LIP) respond to saccadic eye movements, whereas neurons in VIP respond during SPEMs (Andersen, 1997; Colby and Goldberg, 1999). In an early study on visual response properties in monkey PPC, Colby et al. (1993) showed that VIP neurons responded selectively to the direction and speed of moving visual stimuli. Later studies demonstrated that the majority of VIP neurons exhibited direction-selective responses for more complex motion stimuli such as expanding optic flow (Schaafsma and Duysens, 1996; Schaafsma et al., 1997; Bremmer et al., 2002). Similar response properties related to optic flow stimuli were also found in area $7 \mathrm{a}$ on the posterior parietal convexity (Steinmetz et al., 1987; Schaafsma and Duysens, 1996; Siegel and Read, 1997).

Using a memory-guided saccade paradigm, we identified IPS1-IPS4 in human PPC, as described in previous studies (Sereno et al., 2001; Schluppeck et al., 2005; Swisher et al., 2007; Konen and Kastner, 2008). We found two additional topographically organized areas in human PPC, which we will describe here as IPS5 and superior parietal lobule 1 (SPL1). Based on the anatomical location and topographic organization, IPS5 may correspond to the "parietal face area" (Sereno and Huang, 2006). Functional response properties related to eye movements and visual motion were then probed in these six areas. We found a gradual change in the preference for saccades or SPEMs along a posterior-anterior axis in the PPC. All topographically organized areas showed motion-selective responses, as assessed in functional magnetic resonance adaptation (fMR-A) paradigms (GrillSpector et al., 1999), to planar, circular, and radial optic flow patterns. Our results suggest strikingly similar characteristics, but also some differences in the functional organization of human and monkey PPC.

\section{Materials and Methods Subjects}

Six subjects gave informed written consent for participation in the study, which was approved by the Institutional Review Panel of Princeton University. All of the subjects (four men; 24-36 years of age) were in good health with no history of psychiatric or neurological disorders. Subjects had normal or corrected-to-normal visual acuity. All subjects participated in four scanning sessions, two sessions for the localization of topographically organized areas in parietal, frontal, and occipital cortex, and one session each for eye movement and visual motion studies.

\section{Visual display}

The stimuli were generated on a Macintosh G4 computer (Apple Computers) using MATLAB software (The MathWorks) and Psychophysics Toolbox functions (Brainard, 1997; Pelli, 1997). Stimuli were projected from a PowerLite 7250 liquid crystal display projector (Epson) outside the scanner room onto a translucent screen located at the end of the scanner bore. Subjects viewed the screen at a total path length of $60 \mathrm{~cm}$ through a mirror attached to the head coil. The screen subtended $30^{\circ}$ of visual angle in the horizontal dimension and $26^{\circ}$ in the vertical dimension. A trigger pulse from the scanner synchronized the onset of stimulus presentation to the beginning of the image acquisition.

\section{Visual stimuli and experimental design}

\section{Memory-guided saccade task}

A memory-guided saccade task was used to localize topographically organized areas in parietal and frontal cortex (Kastner et al., 2007). The task was performed at eight peripheral locations arranged clockwise around a central fixation point (see Fig. 1a). During each trial of the task, subjects maintained fixation at a central cross while a target stimulus $\left(0.3^{\circ}\right)$ was presented in one of the peripheral locations at $\sim 10^{\circ}$ eccentricity for 500 $\mathrm{ms}$, the location of which had to be remembered, followed by distracters presented for $3 \mathrm{~s}$. The distracters were 100 dots $\left(0.3^{\circ}\right)$ randomly configured within an annulus spanning $9-11^{\circ}$ eccentricity, with a new configuration displayed every $500 \mathrm{~ms}$. The disappearance of the distracters indicated to the subjects to execute a saccade to the remembered target location and then immediately back to fixation $(750 \mathrm{~ms})$. Subjects had another $750 \mathrm{~ms}$ to prepare for the next trial, which started with the appearance of a target at a new location. Each trial at a given target location was $5 \mathrm{~s}$. The peripheral locations were arranged such that the target appeared at 12, 1:30, 3, 4:30, 6, 7:30, 9, and 10:30 o'clock positions. The first target appeared at the right horizontal meridian and subsequent targets were presented in a counterclockwise sequence through eight equally spaced positions. The position of each target was randomly jittered by up to $2.5^{\circ}$ in each direction to reduce the predictability of the task. Each run lasted for $360 \mathrm{~s}$ and was composed of eight $40 \mathrm{~s}$ cycles of the sequence of the eight positions and $20 \mathrm{~s}$ of fixation at the beginning and at the end of each run. Eight runs were performed during a given scanning session. Eye movements were recorded to test the correct performance of the memory-guided saccade task during practice sessions outside the scanner (Applied Science Laboratories). Ilab software was used to analyze the eye movements (Gitelman, 2002). A velocity criterion (three samples $>10 \%$ of saccade maximum) was used for the detection of saccade onset. For SPEMs, the gain, that is, the ratio between target and eye velocity (degrees per second) was calculated to determine the accuracy of the performance.

\section{Retinotopic mapping}

Retinotopic visual field representations were determined with standard phase-encoding analysis techniques to localize visual areas V1, V2, V3, V4, V3A, and V7 (Sereno et al., 1995; Engel et al., 1997; Schneider et al., 2004). The stimulus was a flickering chromatic radial checkerboard $(4 \mathrm{~Hz}$ flicker frequency) with both luminance and chromatic contrast (Swisher et al., 2007). For polar angle mapping, the checkerboard was presented as a rotating wedge. For eccentricity mapping, the checkerboard was presented as a contracting annulus. Each run was composed of 1532 s cycles of a rotating wedge and contracting annulus, respectively, while subjects performed a detection task at central fixation. Four runs were performed during a given scanning session.

\section{Eye movement studies}

The purpose of these studies was to probe responses evoked by saccades and SPEMs in topographically organized areas of parietal and frontal cortex. Subjects performed two types of eye movements in separate scan runs: saccades and smooth pursuit. In both studies, subjects were instructed to track the visual target, which was a black dot $\left(0.2^{\circ}\right)$ presented on a gray background. The dot appeared first at the center of the screen and either jumped with a frequency of $1 \mathrm{~Hz}$ or moved with a velocity of $10 \%$ along one of four axes (horizontal meridian, vertical meridian, diagonal between the top right and the bottom left of the screen, and diagonal between the top left and the bottom right of the screen). For the saccade task, subjects performed visually guided saccadic eye movements to track the jumping target $\left(5-20^{\circ}\right.$; average amplitude of $\left.12.5^{\circ}\right)$. For the smooth pursuit task, subjects performed SPEMs to keep the moving target on the fovea $\left( \pm 10^{\circ}\right)$. To minimize the number of catch-up saccades during the pursuit task, the outline of a circle was centrally presented (diameter, $20^{\circ}$ ), which symbolized the turning points of the moving target. The order of eye movement axes was counterbalanced across scans. Each run lasted for $195 \mathrm{~s}$ and contained six epochs of eye movements, each lasting for $15 \mathrm{~s}$ and alternating with equally long fixation periods. Each run started and ended with central fixation for $15 \mathrm{~s}$ and was repeated four times during a scanning session. Before scanning, each subject was trained in behavioral sessions, in which eye movements were monitored (Applied Science Laboratories) to test the correct performance of both saccade and smooth pursuit tasks (Gitelman, 2002). Supplemental Figure 1 (available at www.jneurosci.org as supplemental material) shows examples of the eye movement recordings.

\section{Visual motion study}

To probe selectivity for visual motion in areas of parietal, frontal, and occipital cortex in humans, we used fMR-A and investigated three con- 
ditions of optic flow stimuli: planar, circular, and radial motion. Planar, circular, and radial motion stimuli are all considered to be associated with the visual impression during self-motion (Duffy, 1998). However, physiology studies in monkeys have established differences in the neuronal representation of optic flow stimuli (Merchant et al., 2001), thereby justifying the separate investigation of the three different motion conditions. One thousand dots were presented within a $15^{\circ}$ diameter circular aperture sparing the central $0.75^{\circ}$ of the visual field. Each $\operatorname{dot}\left(0.15^{\circ}\right)$ moved with an average velocity of $8 \%$ and had a maximum lifetime of $500 \mathrm{~ms}$, after which it was assigned to a new random location within the aperture. If a moving dot traveled outside the aperture, it was relocated to a new random location within the aperture. In the planar motion condition, random dots moved rightward, leftward, upward, or downward. In the circular motion condition, random dots moved clockwise or counterclockwise. In the radial motion condition, random dots moved toward or away from central fixation. During a given run, six epochs of moving dots lasting for $16 \mathrm{~s}$ alternated with equally long presentations of stationary dots. Each run started and ended with the presentation of stationary dots for $16 \mathrm{~s}$ and was repeated six times during a scanning session. The fMR-A paradigm probed two levels of motion adaptation that were distinguished by the number of different motion directions presented in a given epoch (see Fig. 1b). The adapted condition consisted of random dots moving continuously into one direction. The nonadapted condition consisted of random dots moving successively into all eight directions. Each epoch consisting of planar, circular, or radial motion (adapted conditions) as well as each epoch consisting of different motion directions (nonadapted condition) was repeated nine times during a given scanning session. The number and order of specific motion directions were randomized across scans.

To minimize the possibility that differences in activation evoked during adapted and nonadapted conditions were confounded by visual attention, subjects performed a letter detection task at fixation by monitoring a rapid serial visual presentation stream for the occurrence of target letters (A, B, C) among letters, numbers, and keyboard symbols. The presentation duration of each item was $200 \mathrm{~ms}$ with target letters occurring on average every $2.3 \mathrm{~s}$. During training sessions, reaction time (RT) data were collected by requiring subjects to press a button on the appearance of a target letter. During scanning experiments, subjects were instructed to count the letters instead and to report the overall count after each run. The counting task was implemented because higher-order areas of the dorsal visual pathway are known to be associated with the transformation of sensory input to motor output, and therefore a motor response may influence the activity evoked in parietal and frontal cortex, thereby confounding responses related to the visual motion task. Because subjects performed the task throughout the entire experiment, any counting activity was "subtracted out," or alternatively, affected all conditions equally.

\section{Data acquisition}

Data were acquired with a 3 tesla head scanner (Siemens) using a standard head coil (Nova Medical). An anatomical scan [magnetizationprepared rapid-acquisition gradient echo (MPRAGE) sequence; repetition time (TR), $2.5 \mathrm{~s}$; echo time (TE), $4.3 \mathrm{~ms}$; flip angle, $8^{\circ}$; $256 \times 256$ matrix; $1 \mathrm{~mm}^{3}$ resolution] was acquired in each session to facilitate cortical surface alignments. For cortical surface reconstructions, two high-resolution structural scans (same MPRAGE sequence and parameters as above) were acquired in a separate session and averaged.

For all studies, functional images were taken with a gradient echo, echoplanar sequence (motion study: TR, $2 \mathrm{~s}$; TE, $30 \mathrm{~ms}$; flip angle, $90^{\circ}$; all other studies: TR, $2.5 \mathrm{~s}$; TE, $40 \mathrm{~ms}$; flip angle, $90^{\circ}$ ). For the visual motion study, 34 axial slices (slice thickness, $3 \mathrm{~mm}$; gap, $0 \mathrm{~mm}$; voxel size, $3 \times 3 \times$ $3 \mathrm{~mm}^{3}$ ) covering the whole brain were acquired in six series of 104 volumes. For the memory-guided saccade task and the eye movement studies, 25 axial slices (slice thickness, $2 \mathrm{~mm}$; gap, $1 \mathrm{~mm}$; voxel size, $2 \times$ $2 \times 2 \mathrm{~mm}^{3}$ ) covering parietal and frontal cortex were acquired in eight series of 140 volumes, and four series of 78 volumes, respectively. For retinotopic mapping, 25 axial slices (slice thickness, $2 \mathrm{~mm}$; gap, $1 \mathrm{~mm}$; voxel size, $2 \times 2 \times 2 \mathrm{~mm}^{3}$ ) covering the occipital cortex were acquired in four series of 192 volumes. A series of in-plane magnetic field map images were obtained in each scan run to perform echo planar imaging undistortion (field of view, $256 \times 256 \mathrm{~mm}, 128$ matrix; TR, $345 \mathrm{~ms}$; TE, 5.06/8.06 ms; flip angle, $40^{\circ}$; bandwidth, $260 \mathrm{~Hz} /$ pixel).

\section{Data analysis}

Data were analyzed by using AFNI (http://afni.nimh.nih.gov/afni), FREESURFER (http://surfer.nmr.mgh.harvard.edu), and SUMA (http:// afni.nimh.nih.gov/afni/suma). The functional images were motioncorrected to the image acquired closest in time to the anatomical scan (Cox and Jesmanowicz, 1999), and the high-resolution data were undistorted using the field map scan.

\section{Memory-guided saccade task and retinotopic mapping}

Fourier analysis was used to identify voxels activated by the tasks (Bandettini et al., 1993; Engel et al., 1997; Schneider et al., 2004). The ratio of the signal power at the fundamental stimulus frequency and average power at all frequencies was computed, excluding the first and second harmonics and very low frequencies ( $1-3$ cycles/run). Under the assumption of white, temporally uncorrelated noise, the power at each frequency is an independent, identically distributed $\chi^{2}$ random variable. Thus, the resulting ratio of signal power is $F$ distributed. The $p$ values of the activation at each voxel were calculated on the basis of an $F$ statistic. Activation maps were thresholded at $p<0.001$. Although the precise determination of the $p$ values requires assumptions about the properties of the noise in the measurements (Schluppeck et al., 2005), $p$ values present a useful tool for the visualization and estimation of significance of phase maps in mapping studies (Tootell et al., 1997, 1998; Hadjikhani et al., 1998; Schneider et al., 2004; Kastner et al., 2007). To validate these phase maps, we performed complementary analyses including the demonstration of continuous progression of response phases in each area and phase reversals at area boundaries, as described below. To correctly match the phase delay of the time series of each voxel to the phase of the visual stimulus/saccade direction, and thereby localize to the region of the visual field to which the underlying neurons responded best, the response phases were corrected for the hemodynamic lag (3s). Data were spatially smoothed on the surface with a $6 \mathrm{~mm}$ Gaussian kernel.

\section{Eye movement studies}

Square-wave functions matching the functional magnetic resonance imaging (fMRI) time series of the experimental design were convolved with a gamma-variate function and used as regressors of interest in a multiple regression model in the framework of the general linear model (Friston et al., 1995). Additional regressors to account for variance attributable to baseline shifts between time series, linear drifts within time series, and head motion were included in the regression model. Data were spatially smoothed with a $4 \mathrm{~mm}$ Gaussian kernel.

Activated voxels resulting from the comparison between saccades or SPEMs versus fixation periods were assigned to regions of interest (ROIs) (see below for definitions). Statistical maps were thresholded at $p<0.001$ (uncorrected for multiple comparisons). For each subject, the time series of fMRI signals were averaged over all activated voxels in a given ROI and normalized to the mean intensity obtained during the fixation period. All time course analyses were performed on unsmoothed data. To quantify preferential responses evoked by saccades and SPEMs in topographically organized areas of parietal and frontal cortex, we performed the following analyses.

Volumetric analysis. For each subject and ROI, we determined the number of voxels that were activated either during the execution of saccades or SPEMs, or that were commonly activated during the execution of both types of eye movements (at a fixed threshold of $p=0.001$ ). The resulting volumes were normalized to the overall activated volume within an ROI. These normalized volumes were then averaged across all subjects to yield group data. Statistical significance was assessed with a repeated-measures ANOVA followed by a $t$ test.

Mean signal change. The fMRI time series were first averaged across all voxels that were activated during the execution of saccades or SPEMs within a given ROI and then collapsed by eye movement condition. For each subject, the six highest fMRI intensities obtained during each eye movement condition were averaged resulting in mean signal changes, 
which were then averaged across subjects to yield group data. Statistical significance was assessed with paired $t$ tests.

Selectivity index. A selectivity index (SI) was computed to quantify preferential responses evoked by saccades and SPEMs and to compare them across areas $\left[\mathrm{SI}=\left(R_{\text {saccade }}-R_{\mathrm{SPEM}}\right) /\right.$ $\left(R_{\text {saccade }}+R_{\text {SPEM }}\right) ; R_{\text {saccade }}$ represents mean fMRI signals obtained during saccadic eye movements, and $R_{\mathrm{SPEM}}$ represents mean fMRI signals obtained during SPEMs]. Positive index values indicate stronger responses evoked by saccadic eye movements, negative values indicate stronger responses evoked by SPEMs, and values around zero indicate no preference for one or the other eye movement. For each subject, the SI was determined in a given ROI. The SIs were then averaged across subjects to yield group data. Statistical significance was assessed with a bootstrap analysis (Efron and Tibshirani, 1991). The mean fMRI signals evoked during the saccade and smooth pursuit tasks were pooled together across subjects for each ROI. Values were then randomly drawn repeatedly from this pool and assigned as mean fMRI signals for each subject for both eye movement tasks. Using these randomly drawn numbers, the SIs were again calculated separately for each subject and then averaged across subjects. This was repeated 10,000 times to yield a distribution of average SIs for each ROI. Finally, the values obtained from the original data were compared with the 2.5 percentile of each side of the distribution to assess their level of significance. Additional bootstrap analyses were performed to compare the index values across ROIs.

\section{Visual motion study}

For the visual motion study, activated voxels resulting from the comparison between moving dots versus stationary dots were assigned to ROIs. Data were spatially smoothed with a $4 \mathrm{~mm}$ Gaussian kernel. Statistical maps were thresholded at $p<0.001$ (uncorrected for multiple comparisons). For each subject, the time series of fMRI signals were averaged over all activated voxels in a given ROI and normalized to the mean intensity obtained during the presentation of stationary dots. To obtain mean signal changes, the six peak intensities of the fMRI signal during the adapted and nonadapted conditions were averaged. The mean signal changes were then averaged across subjects to yield group data.

Adaptation effects were defined on the basis of a significant response difference between the adapted and nonadapted conditions. An adaptation index $(\mathrm{AI})$ was computed to quantify the adaptation effects and to compare them across areas $\left[\mathrm{AI}=\left(R_{\text {different }}-R_{\text {identical }}\right) /\left(R_{\text {different }}+\right.\right.$ $\left.R_{\text {identical }}\right) ; R_{\text {identical }}$ represents mean fMRI signals obtained during the adapted condition with one motion direction, and $R_{\text {different }}$ represents mean $\mathrm{fMRI}$ signals obtained during the nonadapted condition with eight different motion directions]. Positive index values indicate stronger responses to nonadapted than to adapted conditions and thus an adaptation effect (i.e., the higher the AI, the stronger the adaptation effect). Negative values indicate stronger responses to adapted than to nonadapted conditions, and values around zero indicate the absence of adaptation effects. The AI was determined in a given ROI for each subject. The AIs were then averaged across subjects to yield group data. Statistical significance was assessed with a bootstrap analysis similar to the one described above (Efron and Tibshirani, 1991).

\section{Definition of regions of interest}

\section{Occipital cortex}

Retinotopic mapping was used to identify areas V1, V2, V3, V4, V3A, and V7. We refer to human V4 as an area that contains distinct representations of the contralateral upper and lower quadrants located next to each other medially and laterally on the posterior fusiform gyrus (Kastner et al., 1998; Wade et al., 2002). Area V3A showed a continuous map of the contralateral hemifield and was localized anterior to V3 (Tootell et al., 1997). Area V7 exhibited a representation of the contralateral hemifield and was localized along the transverse occipital sulcus (Press et al., 2001). The location and visual field representation within area V7 was confirmed in the memory-guided saccade paradigm.
The visual motion experiment also served as a localizer for MT+, which was defined as the area localized near the inferior temporal sulcus that responded significantly stronger to presentations of moving dots versus stationary dots ( $t$ test on the contrast moving dots $>$ stationary dots; $p<0.001$ ) (Huk et al., 2002). It should be noted that we did not discriminate between human middle temporal area (MT) and medial superior temporal area (MST) and refer to the motion-sensitive complex as $\mathrm{MT}+$

\section{Parietal and frontal cortex}

Contiguous clusters of activated voxels within parietal and frontal cortex that showed a systematic representation of visual space in the memoryguided saccade task were defined as topographically organized ROIs. In parietal cortex, the memory-guided saccade paradigm revealed six topographically organized areas. The statistical thresholds for vertical meridian representations that form the borders between areas differed between subjects as well as between hemispheres. To account for these differences, the borders were individually drawn along the most significant representations of the upper and lower vertical meridians. Using this procedure, all borders were identified on the basis of three different significance levels. Supplemental Table 1 (available at www.jneurosci.org as supplemental material) gives detailed information about the statistical thresholds of each border in each hemisphere and subject ranging from $p<$ $0.001, p<0.0001$, to $p<0.00001$. The table shows that 75 of 108 borders were highly significant $(p<0.00001)$. To report the Talairach coordinates, the statistical maps and structural images for each subject were transformed into Talairach space (Table 1) (Talairach and Tournoux, 1988). In frontal cortex, a topographic map was found in the superior branch of the precentral sulcus and caudalmost part of the superior frontal sulcus and thus in the region of the frontal eye fields (FEFs), confirming our previous studies (Kastner et al., 2007). The topographic maps in parietal and frontal cortex will be described in Results and are displayed in Figures 2, 4, and 5, and supplemental Figures 2 and 3 (available at www.jneurosci.org as supplemental material).

\section{Results}

We identified six topographically organized areas in PPC and probed their functional characteristics related to the representation of eye movements and visual motion $(n=6)$. In the first series of experiments, neural responses evoked by saccades and SPEMs were investigated. Subjects performed either visually guided saccades or SPEMs alternating with fixation periods. In the second series, neural selectivity to different kinds of visual motion (planar, circular, or radial motion) was examined using an fMR-A paradigm.

\section{Topographically organized areas in parietal and frontal cortex}

The topographic organization of spatial maps in parietal and frontal cortex was investigated using a memory-guided saccade task (Sereno et al., 2001; Kastner et al., 2007). Briefly, subjects performed memory-guided saccades to multiple peripheral locations arranged clockwise around a central fixation point (Fig. 1a). This "cognitive mapping" approach revealed a contiguous band of topographically organized areas in PPC, consisting of five areas along and around the IPS, which we refer to as IPS1-IPS5, and 


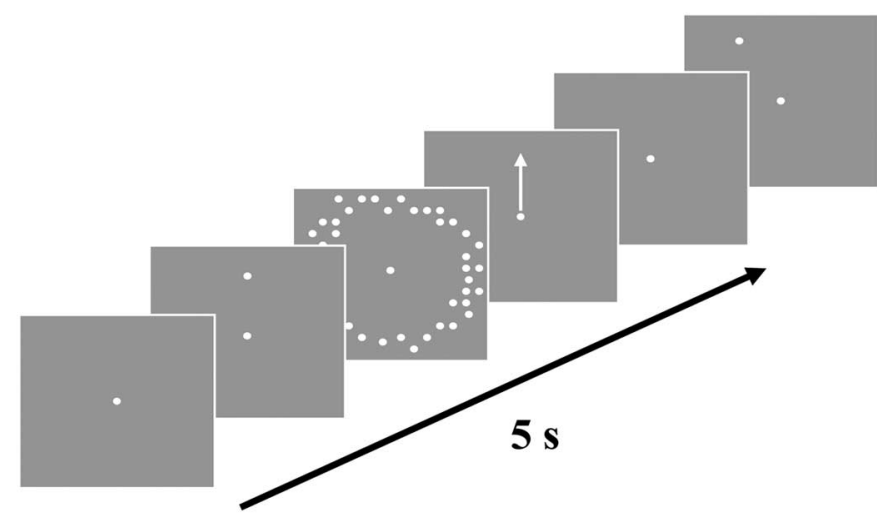

$\mathrm{b}$

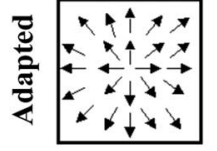

1

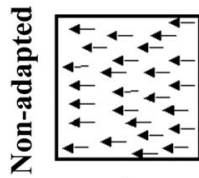

1

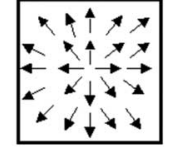

1

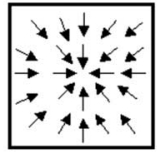

2

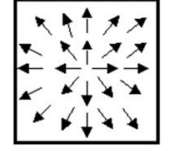

1

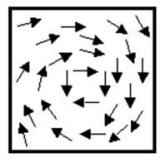

3

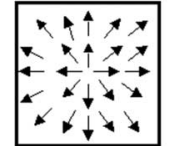

1

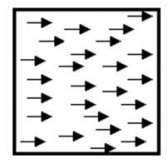

4

\section{\# Different motion directions}

Figure 1. Experimental designs. $\boldsymbol{a}$, Memory-guided saccade task. Subjects performed the task at eight different peripheral locations arranged clockwise around the fixation point. Example of a $120^{\prime}$ clock trial. Subjects maintained fixation throughout the trial. A target stimulus was presented at $\sim 10^{\circ}$ eccentricity for $0.5 \mathrm{~s}$, the location of which subjects had to remember during the following presentation of a ring of distracters for $3 \mathrm{~s}$ at similar eccentricities. Subjects were prompted to saccade to the remembered target location on disappearance of the distracters, as schematically indicated by the arrow. $\boldsymbol{b}$, Examples of motion stimuli and fMR-adaptation paradigm. The visual motion display consisted of random dots moving with fixed velocity about central fixation, in which subjects performed a letter detection task. Planar (rightward, leftward, upward, downward), circular (clockwise, counterclockwise), and radial (expansion, contraction) motion stimuli were tested. The top row illustrates the adapted condition, as exemplified by an expanding stimulus, in which the same stimulus was shown repeatedly (as indicated by " 1 "). The bottom row illustrates the nonadapted condition, in which the random dots traveled successively into eight different directions of motion (as indicated by "1". . . " 8 "; leftward, contracting, clockwise, rightward, and counterclockwise stimuli are shown as examples). The number of identical motion directions presented in a given condition is indicated below the pictures ranging from one in the adapted condition to eight in the nonadapted condition.

one area branching off into the superior parietal lobule, which we refer to as SPL1. Each area contained a representation of the contralateral visual field and was separated toward neighboring areas by reversals in the visual field orientation.

Figure 2 and supplemental Figure 2 (available at www. jneurosci.org as supplemental material) show the topographically organized areas for all subjects (S1-S6). The color code indicates the phase of the fMRI response that corresponds to a given position in the visual field. The responses were lateralized such that the right visual field (red) was represented in the left hemisphere, whereas the left visual field (green) was represented in the right hemisphere. Responses to the upper and lower vertical meridians (blue and yellow) were represented in both hemispheres. Figure 3 shows the volumetric representations of the visual field for the group of subjects $(n=6)$, plotted in polar coordinates for each area. Anterior to visual area V7, two areas were located in the posterior part of the IPS (IPS1, IPS2). The boundary between V7 and IPS1 was formed by the lower vertical meridian (yellow), whereas the boundary between IPS1 and IPS2 corresponded to the representation of the upper vertical meridian (blue). Percentages of $80 \pm 6$ and $78 \pm 7 \%$ of activated voxels responded more

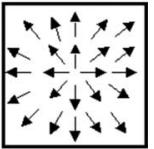

1

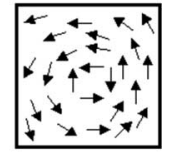

8 strongly to the contralateral side in IPS1 and IPS2, respectively (contralateral vs ipsilateral representation; $p<0.001$ ) (Fig. $3)$. These findings confirm previous reports of topographic organization in IPS1 and IPS2 that were revealed by a memoryguided saccade task similar to ours (Schluppeck et al., 2005), covert shifts of spatial attention (Silver et al., 2005), or retinotopic mapping with a central detection task (Swisher et al., 2007).

Anterior to IPS1 and IPS2, IPS3 and IPS4 were located in the anterior/lateral branch of the IPS. The boundary between IPS2 and IPS3 corresponded to the lower vertical meridian (yellow), whereas IPS3 and IPS4 were bounded by the representation of the upper vertical meridian (blue). Percentages of $86 \pm 7$ and $87 \pm 4 \%$ of activated voxels responded more strongly to the contralateral hemifield in IPS3 and IPS4, respectively (contralateral vs ipsilateral representation; $p<0.001$ ) (Fig. 3). IPS3 and IPS4 have been previously defined using retinotopic mapping with a central detection task (Swisher et al., 2007). Furthermore, the anatomical location, Talairach coordinates, and the representation of the visual field running rostrally from the lower to the upper vertical meridian indicate that IPS3 and the topographically organized area reported by Sereno et al. (2001) may be identical areas.

Anterior and lateral to IPS4, a fifth topographic area was found that we refer to as IPS5. IPS5 extended into the intersection between the IPS and the postcentral sulcus. IPS 4 and IPS5 were bounded by the representation of the lower vertical meridian (yellow). The anterior border of IPS5 was formed by the upper vertical meridian (blue). A percentage of $86 \pm 5 \%$ of activated voxels responded more strongly to the contralateral side of the visual field in IPS5 (contralateral vs ipsilateral representation; $p<0.001$ ) (Fig. 3). The contiguous band of activation along the IPS ended at the anterior border of IPS5, the most anterior of the topographically organized areas in human PPC. Based on the anatomical location and topographic organization, IPS5 may correspond to the "parietal face area" (Sereno and Huang, 2006).

Notably, the traveling wave of activity defining the topographically organized areas depended strongly on the individual anatomy of the PPC and particularly that of the IPS (Fig. 2; supplemental Fig. 2, available at www.jneurosci.org as supplemental material). For example, the left hemispheres of S1, S3, S5, and S6 as well as the right hemispheres of S2 and S5 exhibited a more curved IPS extending onto the lateral surface of the hemispheres, whereas the left hemispheres of S2 and S4 as well as the right hemispheres of S1, S3, S4, and S6 exhibited a less curved IPS. Accordingly, the functional-anatomical characteristics resulted in a very lateral location of IPS3-IPS5 in hemispheres with a more curved IPS and a very anterior location of IPS3-IPS5 in hemispheres with a less curved IPS. Together, the locations of topographically organized areas strongly depended on the individual 


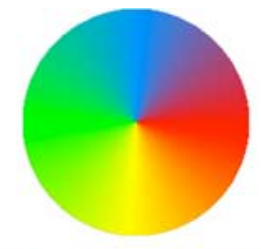

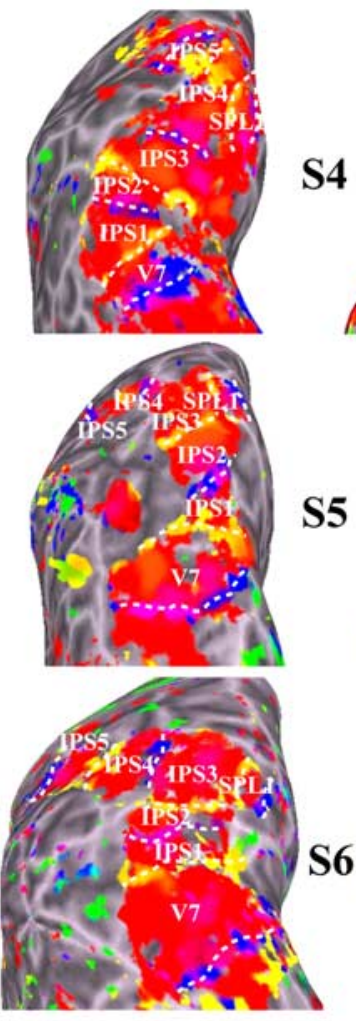

Left hemisphere
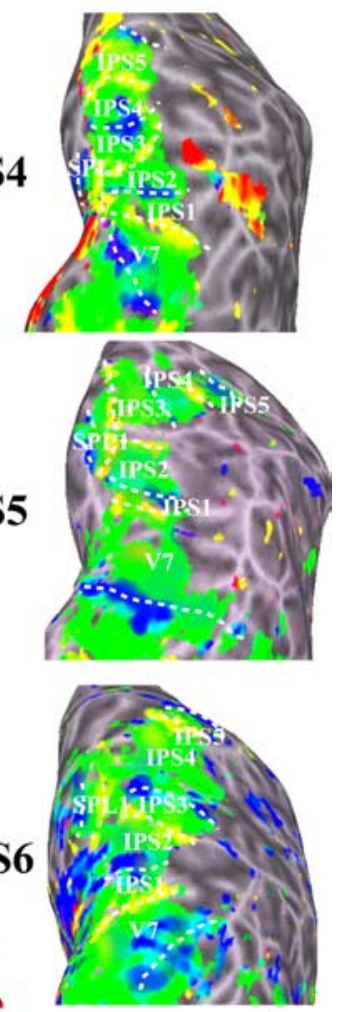

posterior

Right hemisphere

Figure 2. Topographic maps in posterior parietal cortex. Activations obtained from three subjects (S4-\$6) overlaid on inflated left and right hemispheres. The color code is shown for voxels whose responses were correlated with the fundamental frequency of saccade direction indicating the phase of the response $(p<0.001)$. The responses evoked in the memory-guided saccade task were lateralized such that the right visual field was represented in the left hemisphere, whereas the left visual field was represented in the right hemisphere. Area boundaries were formed by the alternating representation of either the upper or the lower vertical meridian.

anatomy of the IPS and are thus highly variable across hemispheres.

An additional representation of the contralateral visual field branched off the medial borders of the most superior IPS subregions and extended into the superior parietal lobule (Fig. 2; supplemental Fig. 2, available at www.jneurosci.org as supplemental material). We refer to this area according to its anatomical location as SPL1. In this area, $89 \pm 3 \%$ of activated voxels responded more strongly to the contralateral hemifield (contralateral vs ipsilateral representation; $p<0.001$ ) (Fig. 3). With respect to the individual anatomy, the most superior IPS areas were IPS1 and IPS2 (right hemisphere: S2, S4), IPS2 and IPS3 (right hemisphere: S1, S3, S5, S6), IPS3 (left hemisphere: S1, S3, S5, S6), or IPS4 (left hemisphere: S2, S4). Notably, the location of the most superior IPS areas was more consistent in the right and more variable in the left hemispheres. Because SPL1 branched off the most superior IPS areas, the anatomical location of SPL1 was also more consistent in the right and more variable in the left hemispheres. SPL1 was characterized by its lateral and medial borders representing the lower and upper vertical meridians (yellow and blue, respectively).

We performed additional analyses to quantify the visual field orientations in each topographically organized area. We focused particularly on IPS5 and SPL1, because data on the topographic layout demonstrating the phase reversals and boundaries of IPS1-IPS4 have already been described in previous studies (Schluppeck et al., 2005; Swisher et al., 2007).

First, we extracted the fMRI time series of small ROIs in SPL1 and IPS5 representing the horizontal and vertical meridians normalized to the mean signal change of each run and averaged across runs. Figure $4 a$ shows the time series for the left hemisphere in one subject. The responses of voxels representing the right horizontal meridian (red), upper vertical meridian (blue), and lower vertical meridian (yellow) were temporally shifted across each cycle following the progression of the visual target "around the clock." The data for the right hemisphere of the same subject are presented as supplemental Figure $3 a$ (available at www.jneurosci.org as supplemental material).

Second, we defined line segments running parallel to and in the middle of the topographically organized areas (indicated by the black rectangular shapes in Fig. 4 and supplemental Fig. 3, available at www.jneurosci.org as supplemental material). The line segments were successively drawn from the posterior border of V7 to the anterior border of IPS5 as well as from the lateral border to the medial border of SPL1. We then calculated the phase values as a function of distance on the flat map for the pixels of each line segment. The origin was defined as the posterior border of V7. The dots in Figure $4 b$ and supplemental Figure $3 b$ (available at www.jneurosci.org as supplemental material) indicate the phase values for each individual pixel. The magenta curves indicate the average phase values as a function of distance. These analyses revealed that each topographically organized area showed a continuous progression of response phases and that a phase reversal occurred at the boundary between two adjacent areas. Both criteria are important features for defining topographically organized areas (Brewer et al., 2002; Schluppeck et al., 2005). This complementary approach confirmed our method for defining topographic areas based on the maps of response phase.

Talairach coordinates and activated volumes of the six parietal areas are given in Table 1. Across subjects, the mean activated volumes were $3375 \pm 1053 \mathrm{~mm}^{3}$ for IPS1, $4023 \pm 1809 \mathrm{~mm}^{3}$ for IPS2, $4212 \pm 729 \mathrm{~mm}^{3}$ for IPS3, $4806 \pm 1215 \mathrm{~mm}^{3}$ for IPS4, $5697 \pm 1323 \mathrm{~mm}^{3}$ for IPS5, and $3348 \pm 1026 \mathrm{~mm}^{3}$ for SPL1. The activated volumes were similar across all topographically organized areas $(p>0.05)$. The statistical significance of the boundaries between these areas differed between subjects as well as between hemispheres and was individually determined. A detailed summary is given in supplemental Table 1 (available at www.jneurosci.org as supplemental material).

In all six subjects, a detailed map of spatial locations was identified in the superior branch of the precentral sulcus and caudalmost part of the superior frontal sulcus and thus in the region of the FEF (Kastner et al., 2007). Figure $5 a$ shows the topography in the region of the FEF for two subjects. In this region, activated voxels coded for saccade directions and memorized locations predominantly in the contralateral hemifield with neighboring saccade directions represented in adjacent locations. It should be noted that there was no continuous retinotopic representation in this region [for more details, see Kastner et al. (2007)]. A percentage of $91 \pm 6 \%$ of activated voxels represented the contralateral 
IPS1

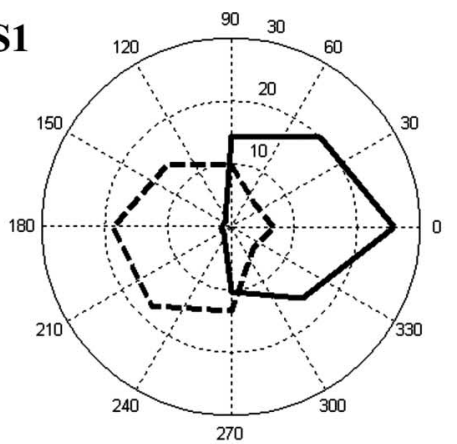

IPS3
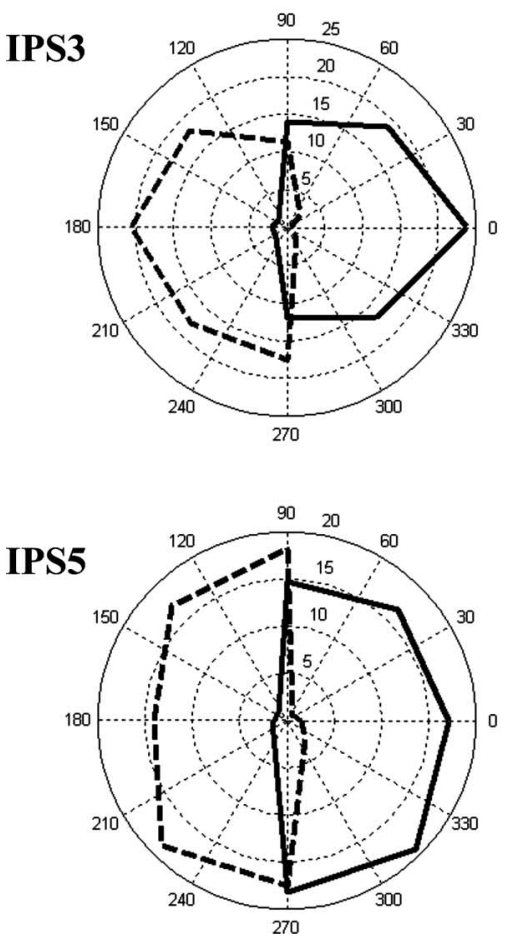

IPS2

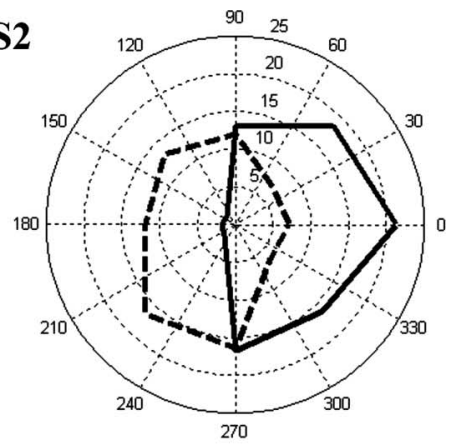

IPS4

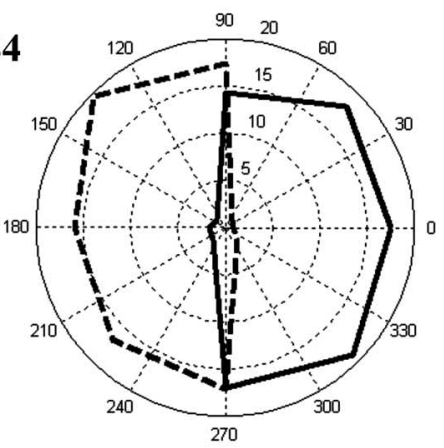

Figure 3. Polar angle representations in IPS1-IPS5 and SPL1. Group analysis of volumetric representations of the visual field in polar coordinates for IPS1-IPS5 and SPL1, plotted for the left (solid lines) and right (broken lines) hemispheres. For each of the eight visual field locations at which the memory-guided saccade task was performed, the percentage of volume was determined for each subject and averaged across subjects to yield group data $(n=6)$. The polar plots showed that all topographically organized areas in PPC represented the contralateral visual hemifield.

hemifield in FEF (contralateral vs ipsilateral representation; $p<$ 0.001). Particular saccade directions were often represented in multiple locations of the map. These results confirmed our previous observations that have been described in detail previously (Kastner et al., 2007).

\section{Representation of saccadic and smooth pursuit eye movements}

In a first set of studies directed at the functional response properties of topographically organized parietal and frontal areas, we probed responses evoked by saccades and SPEMs. For the saccade task, a black dot jumped at a frequency of $1 \mathrm{~Hz}$ and for $5-20^{\circ}$ along one of the cardinal axes. For the smooth pursuit task, the dot target moved with a constant velocity of $10 \%$ along the cardinal axes, and subjects performed SPEMs to track the moving target. For the saccade task, the mean latency for saccadic eye movements was $198( \pm 54)$ ms. For the pursuit task, the gain values for SPEMs were $0.9-1$ indicating an accurate performance of the task. Subjects performed on average $7( \pm 2)$ catch-up sac-

cades per block of SPEMs. Catch-up saccades correct for the position error that accumulates during smooth pursuit tracking when the gain is $<1.0$ and thus could not be avoided in the smooth pursuit task. The average amplitude of the catch-up saccades was $<1^{\circ}$ and thus smaller compared with the average amplitude of the visually guided saccades during the saccade task $\left(12.5^{\circ}\right)$, in which 15 saccades were performed during each block.

As shown in numerous fMRI studies, the execution of both saccades and SPEMs evoked strong activation in parietal and frontal cortex (Pierrot-Deseilligny et al., 1995; Luna et al., 1998; Berman et al., 1999; Brown et al., 2004; Konen et al., 2004, 2005). Here, activated voxels were specifically assigned to areas IPS1-IPS5, SPL1, and FEF based on the contrast between eye movement tasks versus fixation at a conservative threshold of $p<0.001$. Figure 6 and supplemental Figure 4 (available at www.jneurosci.org as supplemental material) depict activations related to the execution of saccades and SPEMs relative to the topographic areas in human PPC for the same subjects shown in Figure 2 and supplemental Figure 2 (available at www. jneurosci.org as supplemental material) $(p=0.00001)$. We compared activation patterns at a fixed threshold that were either evoked during saccadic eye movements (yellow), or evoked during SPEMs (blue), or commonly activated during both types of eye movements (magenta). Although the overlapping activations were widely distributed across the PPC, a systematic pattern of activation regarding the preferential responses evoked by one or the other eye movement was found. When moving from the posterior to the anterior subdivisions of the PPC, the preference in responses gradually changed from saccades to SPEMs. In all subjects, patches of activations that were related to the execution of saccadic eye movements were most often found in IPS1-IPS2 and SPL1. Moreover, in each subject, we found that the most medial activation in SPL1 was preferentially activated by saccadic eye movements. In contrast, patches of activations that were mainly related to SPEMs were most often found in IPS3-IPS5 (left hemisphere: S4, S6; right hemisphere: $\mathrm{S} 1, \mathrm{~S} 3, \mathrm{~S} 5, \mathrm{S6})$.

In frontal cortex, we found a characteristic pattern of representations of saccades and SPEMs in all subjects (exemplified for two subjects in Fig. 5b). In confirmation of previous neuroimaging studies (Petit et al., 1997; Rosano et al., 2002), two eye movement-specific subregions were found in human FEF, which were activated during the execution of either saccades or SPEMs and flanking an interpatched area of overlapping activation. The subdivision related to saccadic eye movements was located in anterior/superior FEF, whereas the subdivision related to SPEMs was located in posterior/inferior FEF. Similar to these findings, monkey physiology and microstimulation studies have shown 


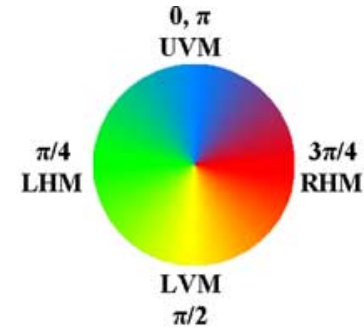

a
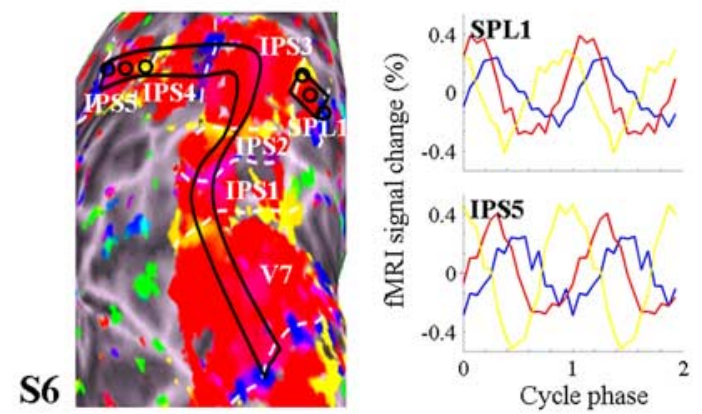

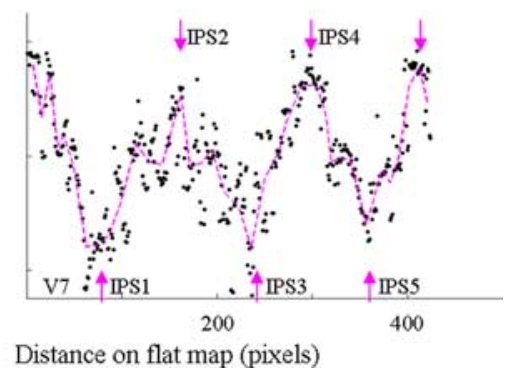

Distance on flat map (pixels) a

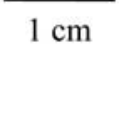

S1
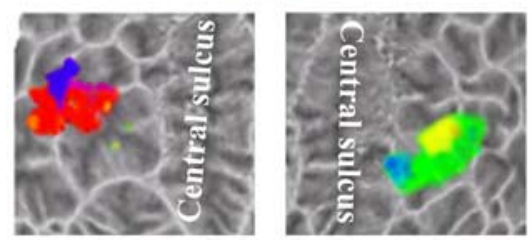

S2
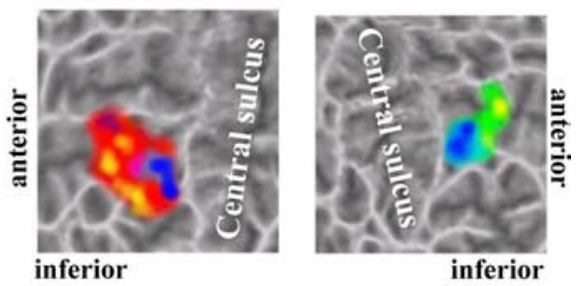

b
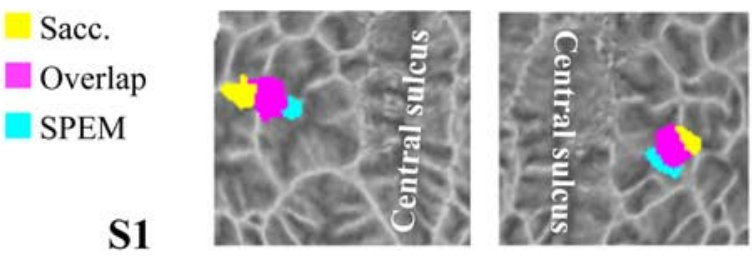

S2

Figure 4. Analysis of topographically organized areas in left PPC. Results for subject $\mathrm{S6}$ are shown. $\boldsymbol{a}$, Time series of fMRI signals from voxels representing the right horizontal meridian (red), upper vertical meridian (blue), and lower vertical meridian (yellow) in SPL1 and IPS5. The time series were averaged across repeated cycles and scans. The three ROls of each area are indicated by the black circles depicted on the surface map. The time series exhibited distinct response phases, allowing the voxels to be reliably assigned to different regions of the visual field. UVM, Upper vertical meridian; RHM, right horizontal meridian; LVM, lower vertical meridian; LHM, lower horizontal meridian. $\boldsymbol{b}$, Response phase as a function of distance on the surface. Small line segments were drawn running parallel to and in the middle of the topographically organized areas as outlined with the rectangular shapes. The line segments were successively drawn from the posterior border of V7 to the anterior border of IPS5 as well as from the lateral border to the medial border of SPL1. The dots indicate the phase values for individual pixels located along the line segments. The magenta curves indicate the average phase values as a function of distance. The results showed the smooth progression of phase values, corresponding to different target locations in the visual field, as a function of distance on the map and most importantly the phase reversals at the shared boundaries between two areas.

that FEF is subdivided into saccade- and SPEM-related areas, located in the anterior bank (Robinson and Fuchs, 1969; Bruce et al., 1985; Huerta et al., 1986; Stanton et al., 1988) and the fundus/ posterior bank of the arcuate sulcus (Gottlieb et al., 1993), respectively. Thus, the comparison between both species revealed similar principles of functional organization in FEF.

To quantify the preferred responses evoked by saccades and SPEMs in topographically organized areas of PPC, we performed a number of additional analyses. First, we determined the number of voxels that were activated during the execution of saccades or SPEMs, or commonly activated during both types of eye movements in each area. Second, we extracted the mean signal changes of fMRI signals across all activated voxels in a given area evoked during the execution of saccades and SPEMs, which in-

inferior

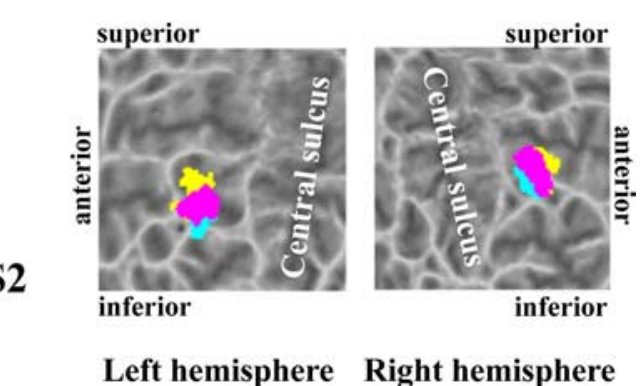

Figure 5. Topographic maps and eye movement representation in frontal cortex. $\boldsymbol{a}$, A topographic map in frontal cortex was found in FEF (i.e., the superior branch of precentral cortex encroaching into the caudalmost part of the superior frontal sulcus). The spatial map of saccade directions represented predominantly the contralateral visual hemifield, as shown overlaid on the flattened left and right hemispheres of two subjects $(\mathrm{S1}, \mathrm{S2})$. The color code is shown for voxels whose responses were correlated with the fundamental frequency of the saccade direction indicating the phase of the response $(p<0.001)$. The visual field color legend labels the saccade direction within the visual field to which each voxel was most responsive. $\boldsymbol{b}$, Eye movement-related activations were thresholded at $p=0.001$. The yellow regions denote voxels that were activated during the execution of saccadic eye movements (Sacc.), the blue regions denote those that were activated during the execution of SPEMs, and the magenta regions denote those that were activated during both types of eye movements. Note that saccade-related activation was located more anterior and superior to SPEM-related activation.

forms about responses evoked by one or the other eye movement in the population response of a given area. And third, we calculated a selectivity index based on the mean signal changes, which permits a comparison of response preferences across areas. It should be noted that the second and third analyses consider only the population response of an area as a whole, thereby discarding regional differences within an area.

For each subject and area, the volumes that were either activated during the execution of saccades or SPEMs, or commonly activated during the execution of both types of eye movements 


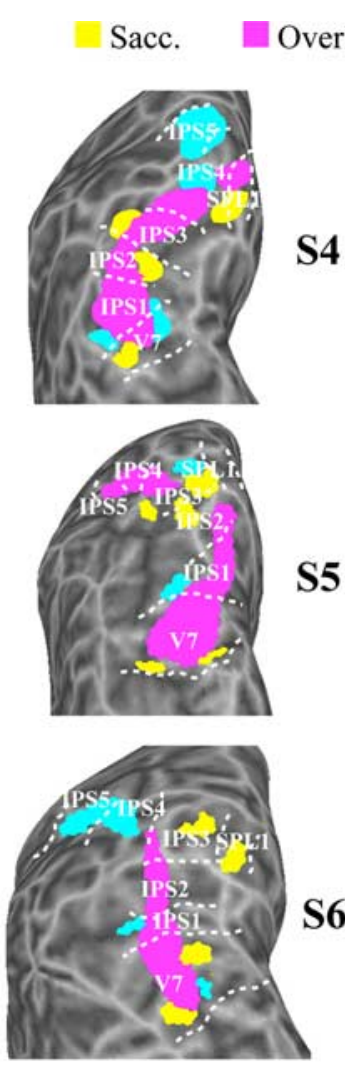

Left hemisphere
S4

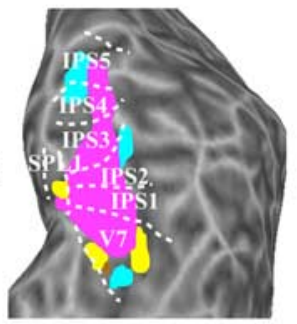

S5

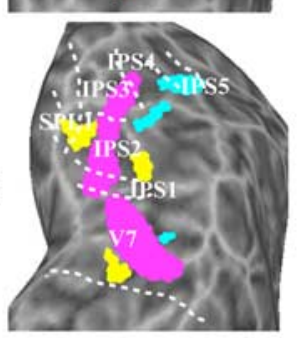

S6

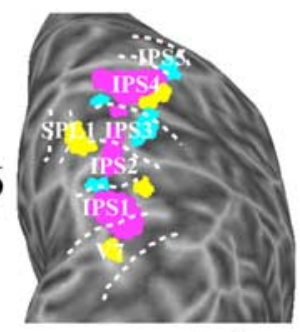

posterior
Right hemisphere

Figure 6. Eye movement representation in posterior parietal cortex. Shown are the same subjects as in Figure 2. Eye movement-related activations were thresholded at $p=0.00001$. The yellow regions denote voxels that were activated during the execution of saccadic eye movements (Sacc.), the blue regions denote those that were activated during the execution of SPEMs, and the magenta regions denote those that were activated during both types of eye movements. When moving from the posterior to the anterior subdivisions of the PPC, the preferential responses gradually changed from saccades to SPEMs. In contrast, the voxels that were activated during both types of eye movements are evenly distributed across all subdivisions.

were determined. The resulting volumes were normalized to the overall volume activated by eye movements within an area and then averaged across subjects. They are plotted as average percentage of activated volume in Figure $7 a$. A repeated-measures ANOVA revealed that the majority of voxels in areas V7, IPS1, IPS4, and FEF were activated during the execution of both types of eye movements $(p<0.01)$. The comparison between the volume that was activated during the performance of one or the other eye movement, however, revealed that the two types of eye movements were differentially represented across areas. The percentage of activated volume in visual area $\mathrm{V} 7$ was similar during the execution of saccades and SPEMs (paired $t$ test, $p>0.05$ ). IPS1 and IPS2 in the posterior part of the IPS and SPL1 in the superior parietal lobule exhibited a significant bias for saccadic eye movements $(p<0.01)$. In contrast, IPS3-IPS5 in the anterior part of the IPS contained significantly more voxels that were activated during the performance of SPEMs $(p<0.01)$. FEF did not show a significant difference in volumes activated during the execution of saccades or SPEMs $(p>0.05)$.

These results were confirmed by an analysis of the mean signal changes that was based on the overall volume activated during the execution of saccades or SPEMs in a given area (Fig. 7b). Visual a

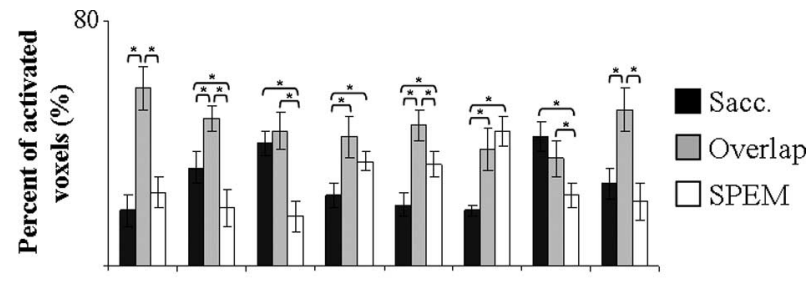

b
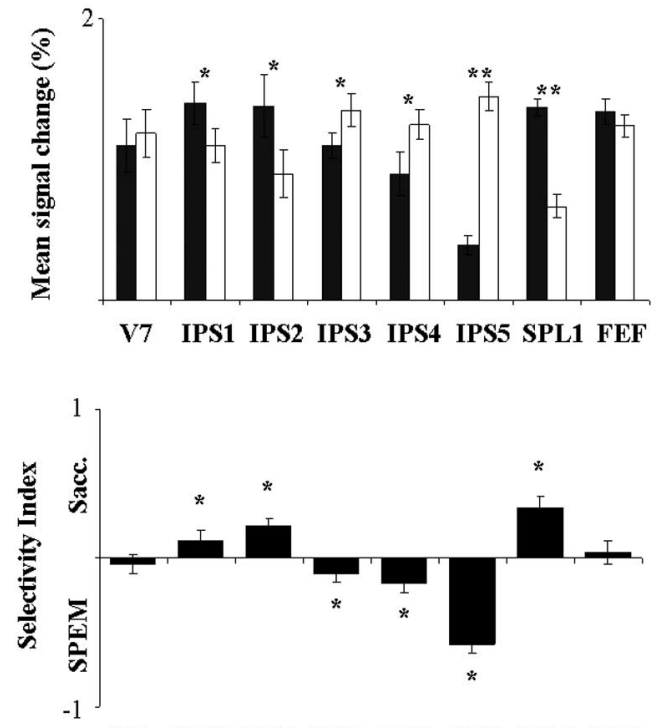

Figure 7. Responses during saccades and SPEMs in posterior parietal cortex. $\boldsymbol{a}$, Volumetric analysis. Number of voxels showing greater responses evoked by saccadic eye movements (Sacc.) (black), SPEMs (white), or both types of eye movements (gray) in areas of PPC and in FEF, averaged across subjects $(n=6)$. *Significant volumetric differences, $p<0.05$. $\boldsymbol{b}$, Mean signal change. Mean fMRI signals were averaged across subjects $(n=6)$ for each area and each eye movement task. For each subject, mean signal change was defined as the average of the six highest intensities of the fMRI signal obtained during saccades (black) or SPEMs (white). * Significant response differences between both tasks ( $\left.p<0.01 ;{ }^{* *} p<0.001\right)$. IPS1-IPS2 and SPL1 showed a significant bias for saccadic eye movements, whereas IPS3-IPS5 showed a significant bias for SPEMs. c, Selectivity index. An SI was defined based on mean signal changes evoked by saccade and smooth pursuit tasks (see $\boldsymbol{b}$ ). Positive index values indicated stronger responses to saccades, negative values indicated stronger responses to SPEMs, and values around zero indicated no preferential responses during one or the other eye movement. Index values that were significantly different from zero were marked by asterisks ( $p<0.05)$. The comparison of index values across areas revealed that SPL1 exhibited the strongest selectivity for saccadic eye movements, whereas IPS5 exhibited the strongest selectivity for SPEMs. Error bars indicate SEM.

area $\mathrm{V} 7$ and FEF showed no significant differences in mean signal changes between saccades and SPEMs (paired $t$ test, $p>0.05$ ). IPS1 and IPS2 showed significantly stronger responses during the performance of saccades compared with SPEMs $(p<0.01)$. In SPL1, the performance of saccades evoked even twice as much signal than SPEMs $(p<0.001)$. The reversed pattern of responses was observed in the anterior subregions of the IPS. IPS3 and IPS4 showed significantly stronger responses during the performance of SPEMs compared with saccadic eye movements $(p<0.01)$. The greatest difference in signal strength was found in IPS5. The performance of SPEMs evoked three times stronger responses compared with saccadic eye movements $(p<0.001)$.

To compare the preferences in responses evoked by saccades and SPEMs across areas, a SI was computed, which estimates the difference in responses evoked during the execution of both eye movement types (Fig. 7c). Positive index values indicate stronger 
responses during the performance of saccades, negative values indicate stronger responses during the performance of SPEMs, and values around zero indicate no preferences for one or the other eye movement. Statistical significance was assessed with a bootstrap analysis. This analysis confirmed the lack of preferred responses evoked by saccades or SPEMs in visual area V7 and FEF in the population response with an index value not different from zero $(p>0.05)$. In contrast, the SIs were found to be significantly different from zero in SPL1 and IPS1-IPS5. IPS1-IPS2 and SPL1 showed a significant bias for saccades $(p<0.05)$. The SI in SPL1 was significantly greater than in IPS1 and IPS2 $(p<0.01)$. IPS3IPS5 exhibited a significant bias for SPEMs $(p<0.05)$. The index value in IPS5 was significantly stronger than those in IPS3 and IPS4 $(p<0.01)$.

Together, the complementary analyses of volume, mean signal changes, and SI revealed that preferences in responses evoked by saccades and SPEMs gradually changed when moving from posterior to anterior/lateral along the IPS. IPS1, IPS2, and SPL1 exhibited preferential responses evoked by saccadic eye movements, whereas IPS3, IPS4, and IPS5 exhibited preferential responses evoked by SPEMs. In addition, the SIs revealed that SPL1 compared with IPS1 and IPS2 showed the strongest bias for saccades, whereas IPS5 compared with IPS3 and IPS4 showed the strongest bias for SPEMs.

We considered the possibility that differences in activation patterns during saccades and SPEMs in PPC might have been confounded by differences in optic flow (i.e., fast retinal image motion during the performance of saccades and slow image motion during the performance of SPEMs). Changing retinal images, however, are unlikely to explain our results of gradient representations of eye movements along the IPS and distinct subregions in the FEF for saccadic and smooth eye movements. First, we performed the visual motion study, described below (see Responses to visual motion), in the same subjects, and, based on the assumption of an optic flow account, one would predict a similar gradient across the IPS and FEF, as seen with the eye movement studies, particularly for planar motion. Instead, all topographically organized IPS areas responded similarly to visual motion including planar motion, whereas the FEF responded generally poorly to optic flow patterns (for additional details, see below, Responses to visual motion). Second, our results are very similar (particularly with respect to FEF) to results obtained in nonhuman primates. Although it may be argued that similar criticism applies to those studies, many of them have used in addition to visually guided eye movements electrical microstimulation to confirm the selectivity of FEF or IPS neurons for saccadic or SPEMs (Robinson and Fuchs, 1969; Bruce et al., 1985; Huerta et al., 1986; Stanton et al., 1988; Gottlieb et al., 1993). And these two approaches yielded converging results. For these reasons, the differential pattern of activation during the saccade and smooth pursuit tasks observed in our studies were likely driven by the execution of both types of eye movements per se rather than differences in optic flow velocities.

\section{Responses to visual motion}

In a second set of studies, we probed another classical response property of dorsal stream areas, that is, responses to visual motion. Neural selectivity for visual motion was tested in parietal, frontal, and occipital cortex using an adaptation paradigm. fMR-A has become a frequently used tool to go beyond the spatial resolution limitations of conventional fMRI to probe neural selectivity in specific cortical areas (Grill-Spector et al., 1999). $\mathrm{fMR}-\mathrm{A}$ is a robust phenomenon in which repeated presentations a

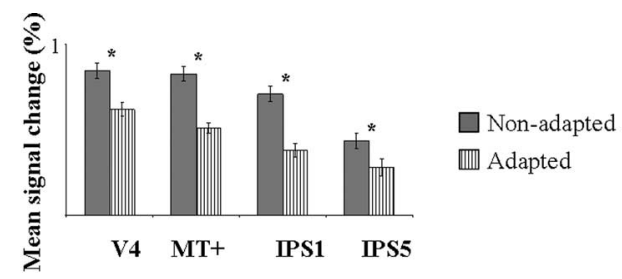

b
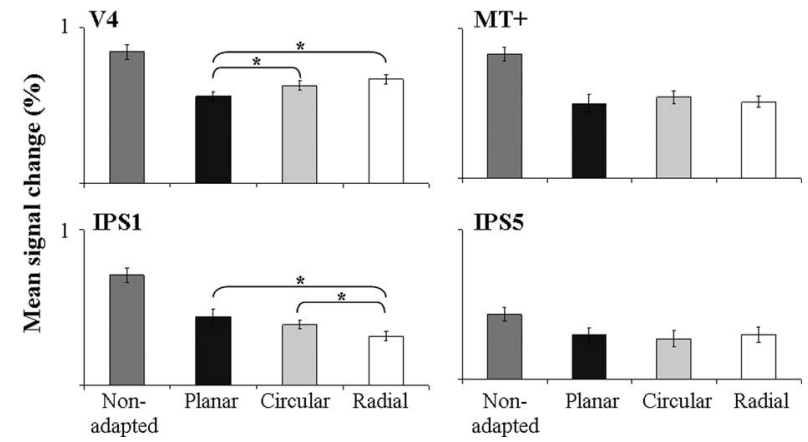

Figure 8. Mean signal changes in V4, MT+, IPS1, and IPS5. Mean fMRI signals were averaged across subjects $(n=6)$ for each area and each condition. For each subject, mean signal change was defined as the average of the six highest intensities of the fMRI signal obtained during the presentation of motion stimuli in the nonadapted condition (dark gray) and in the adapted condition. $\boldsymbol{a}$, The adapted condition was averaged across planar, circular, and radial motion. *Significant response differences between the nonadapted and adapted conditions, $p<0.05$. $\boldsymbol{b}$, The adapted condition is shown separately for planar motion (black), circular motion (light gray), and radial motion (white). * Significant response differences between the adapted conditions, $p<0.05$. Error bars indicate SEM.

of the same visual stimulus leads to response reductions. Dynamic displays of planar, circular, and radial motion were created from random dot displays. For planar motion, random dots moved rightward, leftward, upward, or downward. For circular motion, random dots moved clockwise or counterclockwise. And for radial motion, random dots moved toward or away from central fixation. The motion displays were probed in two different adaptation conditions (Fig. 1b), while subjects performed a demanding letter detection task at fixation. In the adapted condition, random dots moved continuously into one direction. In the nonadapted condition, random dots moved successively into different directions. The nonadapted condition served as a baseline with which all other conditions were compared.

We identified motion-selective areas in parietal, frontal, and occipital cortex by contrasting the adapted versus nonadapted conditions regardless of motion type. Thus, the adapted conditions for planar, circular, and radial motion were combined in this first part of the analysis. We predicted that areas containing large motion-selective neural populations will show response reductions, or adaptation effects, when random dots move continuously into one direction relative to when they move into different directions. In contrast, areas that contain only a small fraction of motion-selective neurons will respond similarly in the adapted and nonadapted conditions and thus show little or no adaptation effects.

Significant adaptation effects were found in each topographic area under consideration including V1, V2, V3, V4, V3A, MT+, V7, IPS1-IPS5, SPL1, and FEF ( $p<0.01)$. Mean signal changes evoked by the adapted and nonadapted conditions (collapsed across the different motion types) are shown for areas V4, MT+, IPS1, and IPS5 in Figure $8 a$. For example, in V4, the mean signal change during the adapted condition induced a response reduction of $26 \%$ compared with the nonadapted condition, indicating 


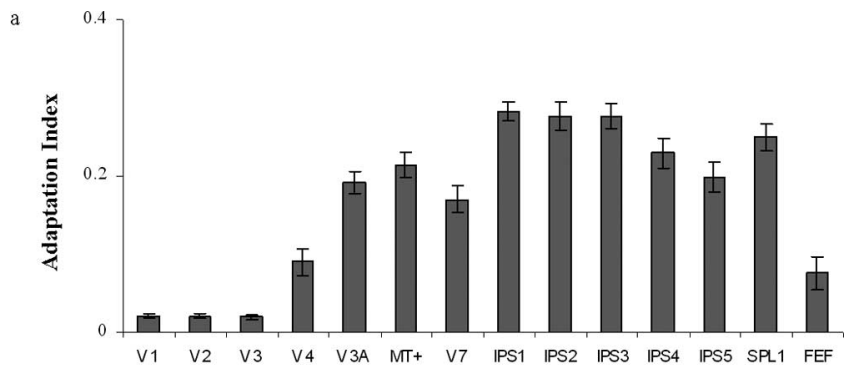

b

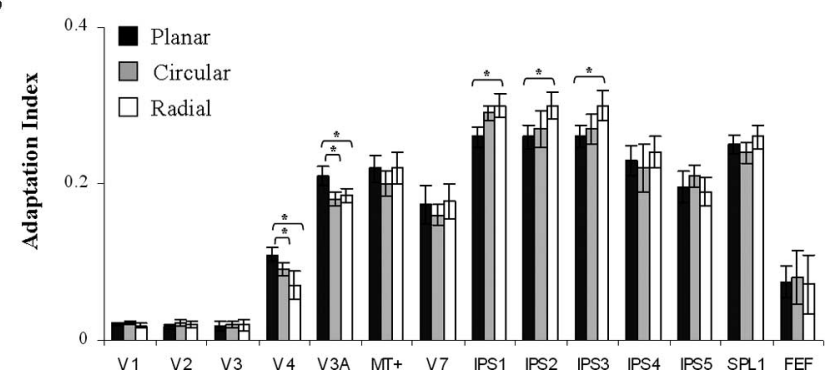

Figure 9. Adaptation index. An Al was defined based on mean signal changes evoked by optic flow patterns presented in the adapted conditions versus the nonadapted condition. Positive index values indicated stronger responses to nonadapted than to adapted conditions, negative values indicated the opposite, and values around zero indicated the absence of adaptation effects. $\boldsymbol{a}$, The adapted conditions were averaged across planar, circular, and radial motion. Note that each area showed stronger responses to nonadapted than to adapted conditions and thus an adaptation effect induced by visual motion. $\boldsymbol{b}$, The adapted conditions were split into planar (black), circular (gray), or radial motion (white). Higher index values indicated stronger adaptation effects. *Significant differences in index values across the different optic flow patterns in a given area, $p<0.05$. V4 and V3A showed stronger selectivity for planar compared with circular and radial motion, whereas IPS1-IPS3 showed stronger selectivity for radial compared with planar motion. Error bars indicate SEM.

an adaptation effect and thus motion selectivity in this area. In IPS1, the mean signal change during the adapted condition was even stronger evoking a response reduction of 54\% compared with the nonadapted condition.

To compare the relative strength of the adaptation effects across areas, the adaptation effects were quantified by an AI. Positive index values indicate stronger responses to nonadapted than to adapted conditions, negative values indicate the opposite, and values around zero indicate the absence of adaptation effects. Statistical significance was assessed with a bootstrap analysis.

This analysis confirmed that all areas along the visual hierarchy showed motion selectivity with index values significantly greater than zero $(p<0.05)$ (Fig. 9a). The comparison between areas revealed that the adaptation effects in early visual areas V1, $\mathrm{V} 2$, and V3 were significantly smaller than in V4 and FEF $(p<$ 0.05). The adaptation effects in the latter areas were significantly smaller compared with the intermediate areas of the dorsal visual pathway V3A, MT + , and V7 $(p<0.05)$. The strongest adaptation effects in the visual system were found in the topographically organized areas of the PPC $(p<0.01)$ with greatest index values in IPS1, IPS2, and IPS3.

Monkey physiology studies have shown that neurons in the intermediate area MT of the visual hierarchy respond primarily to planar motion (Maunsell and Van Essen, 1983; Albright, 1984), whereas neurons in MST and PPC respond to more complex optic flow patterns such as expansion and contraction (Graziano et al., 1994; Schaafsma and Duysens, 1996; Siegel and Read, 1997). To probe for similar distinctions in humans, we examined
fMRI signals evoked by planar, circular, and radial motion separately. Furthermore, this analysis allowed us to investigate the preference for a particular motion type in areas along the visual hierarchy. We hypothesized that areas containing neural populations tuned to a specific type of motion such as planar, circular, or radial motion will show stronger adaptation effects induced by this very type of motion compared with other types of motion. In contrast, areas that contain neuronal populations without preference for a specific motion type will respond similarly across all motion directions.

Mean signal changes evoked by the different motion types in the adapted condition relative to the nonadapted condition are shown for areas V4, MT+, IPS1, and IPS5 in Figure $8 b$. For example, in V4, the response induced by planar motion $(0.56 \%)$ was significantly decreased compared with circular and radial motion ( 0.63 and $0.67 \%$, respectively; $p<0.01$ ) (Fig. $8 b$ ). This result indicates that a substantial amount of neurons appears to be selective for planar motion in area V4. In contrast, in IPS1, radial motion evoked a decreased response $(0.31 \%)$ compared with planar and circular motion $(0.44$ and $0.39 \%$, respectively; $p<0.01$ ) (Fig. 8b). This result suggests that a larger proportion of neurons in IPS1 respond selectively to radial motion than to planar, or circular motion. In the human motion complex MT+, however, the responses induced by planar, circular, and radial motion were similar $(0.5,0.54$, and $0.51 \%$, respectively; $p>0.05)$ (Fig. $8 b$ ), suggesting no preference for a particular motion type.

We determined the AIs for each motion type in a given area and compared the relative strength of adaptation effects across planar, circular, and radial motion (Fig. 9b). This analysis informs about the differential responses evoked by these motion types across the visual system. The results showed that early visual areas V1, V2, and V3 had similar index values for all motion types and thus lacked preference for a particular motion type $(p>$ 0.05 ) (Fig. 9b). Area V4 in the ventral pathway revealed significantly stronger adaptation effects induced by planar motion compared with circular and radial motion $(p<0.05)$ (Fig. 9b). Area V3A in the dorsal pathway showed a similar response profile; planar motion induced stronger adaptation effects compared with circular and radial motion $(p<0.05)$ (Fig. $9 b)$. In contrast, area $\mathrm{MT}+$ in the dorsal pathway showed similar adaptation effects for all motion types tested and thus exhibited no preference for a particular motion type $(p>0.05)$. Physiology studies in nonhuman primates have shown that MT neurons respond primarily direction-selective for planar compared with circular and radial optic flow (Lagae et al., 1994), whereas MST neurons respond preferentially to circular or radial motion (Saito et al., 1986; Duffy and Wurtz, 1991; Graziano et al., 1994). As noted previously, we did not differentiate between MT and MST in our study. Thus, collapsing data across both subdivisions of the human motion complex likely averaged out any preference in MT and MST for a particular motion type. In V7, we found similar adaptation effects across motion types $(p>0.05)$ (Fig. $9 b)$. The topographically organized areas along the posterior IPS, however, showed significant response differences induced by the optic flow patterns. IPS1-IPS3 showed stronger adaptation effects to radial motion than to planar motion $(p<0.01)$ (Fig. 9b). In contrast, SPL1, IPS4-IPS5 in the anterior part of the IPS, and FEF did not show any preference for a particular motion type and thus similar adaptation effects for all optic flow patterns $(p>0.05)$ (Fig. 9b).

To control for the possibility that the adaptation effects found in our study were confounded by visual attention, subjects performed a demanding letter detection task at fixation. They were 
instructed to detect target letters presented in a rapid stream of letters, digits, and keyboard symbols at fixation. The fixation task was designed to help subjects maintaining central fixation and to prevent subjects from covertly attending to the visual motion stimuli. During behavioral sessions outside the scanner, subjects were instructed to press a button on the appearance of a target letter. The efficacy of the detection task was assessed by analyzing performance in the fixation task as a function of presentation condition. For the adapted conditions, the average RT for planar, circular, and radial motion was $410 \pm 45,433 \pm 62$, and $421 \pm 38$ $\mathrm{ms}$, respectively. For the nonadapted condition, the average RT was $402 \pm 54 \mathrm{~ms}$. For the presentation of stationary dots, the average RT was $392 \pm 47 \mathrm{~ms}$. The results showed that RTs were similar across all conditions (repeated-measures ANOVA, $p>$ 0.05). Because the higher-order areas of the dorsal pathway are associated with the transformation of sensory input to motor output, and a motor response may therefore influence the activity evoked in PPC, subjects were instructed to count the letters and report the number after each scan during subsequent scanning sessions and thus did not execute a motor response. The results showed that subjects performed the task with high accuracy ( $89 \pm 8 \%$ correct responses). The RTs and the accuracy data suggest that attention was evenly distributed across the adapted and nonadapted conditions. Furthermore, the differential representation of motion signals with preference for planar motion in area V4 and preference for radial motion in areas IPS1-IPS3 are difficult to reconcile with an account of attentional top-down modulation.

Together, the visual motion studies demonstrated motionselective responses in multiple areas of parietal, frontal, and occipital cortex. The normalized index values indicate that the strength of selectivity was hierarchically organized. Early visual areas showed the weakest adaptation effects, intermediate areas along both the ventral and dorsal pathways showed intermediate adaptation effects, and topographically organized areas in PPC (at the top of the visual hierarchy) revealed the strongest adaptation effects. Furthermore, IPS1-IPS3 showed preference for radial motion over planar motion. This result parallels monkey physiology showing that PPC neurons respond more strongly to more complex optic flow patterns (Schaafsma and Duysens, 1996; Siegel and Read, 1997).

\section{Discussion}

Using a memory-guided saccade paradigm in human PPC (Sereno et al., 2001), we identified six topographically organized areas, referred to as IPS1-IPS5 and SPL1. The identification of a multitude of different areas in human PPC in individual subjects permits pursuing an ROI approach in the study of their response properties and thus extend pioneering studies in this field that used group analyses (Bremmer et al., 2001; Grefkes et al., 2002) (for review, see Grefkes and Fink, 2005). Ultimately, the ROI approach will help reveal not only the functional organization of human PPC but also possible functional homologies to physiologically and anatomically characterized areas in monkey PPC. The present study presents a small step toward such a goal.

From physiology studies, it is not clear whether a topographic large-scale organization similar to the one described here exists in monkey PPC. Physiology studies in LIP and an optical imaging study in area 7 a revealed that the visual receptive fields of neurons in these areas are topographically organized (Blatt et al., 1990; Ben Hamed et al., 2001; Siegel et al., 2003). For example, the visual field in LIP appears to be represented from anterior to posterior with a gradient from the lower to the upper vertical meridian, similar to the visual field representation found in IPS2 and IPS4. Relatively little work has been done to characterize the topographic organization of other areas along the IPS. Because of the differences in spatial scale between functional neuroimaging and single-cell physiology, it is difficult to make inferences about functional PPC homologies solely on the criterion of topographic organization. Thus, it is necessary to probe functional characteristics at the population level in human PPC and to compare them with physiology results from monkey PPC, as was done here in two sets of studies.

First, we probed neural responses evoked by saccades and SPEMs in topographically organized areas of human PPC. The preferential responses during one or the other eye movement changed gradually across areas of the IPS with IPS1-IPS2 and the medial SPL1 preferring saccadic eye movements and IPS3-IPS5 preferring SPEMs. Interestingly, areas in close anatomical proximity such as IPS1-IPS2 and SPL1 in the posterior/medial PPC and IPS3-IPS5 in the anterior/lateral PPC showed similar response characteristics.

This principle of functional organization is similar to the one in nonhuman primates, in which boundaries between areas in PPC are blurred leading to systematic shifts of response characteristics from one area to the next and thus several functional gradients along the IPS (Colby and Duhamel, 1996). For example, VIP, LIP, and area 7a are reciprocally connected and adjacent areas along the IPS (Van Essen et al., 1990). LIP, which is located in the lateral bank of macaque IPS, and area 7a, which is located on the dorsal surface of the same sulcus, have been shown to be involved in the encoding of saccadic eye movements (Andersen et al., 1990). In fact, significantly more neurons in LIP show presaccadic and perisaccadic responses compared with area $7 \mathrm{a}$ (49$63 \%$ in LIP vs $44 \%$ in area 7a) (Barash et al., 1991). In contrast, little evidence for saccade-related activity has been found in VIP, which is located in the fundus of the IPS (Schaafsma and Duysens, 1996) (but see Thier and Andersen, 1998). The majority of VIP neurons responds during SPEMs (53\%) (Schlack et al., 2003 ) and thus a higher proportion than has been reported in LIP (39\%) and area 7a (42\%) (Colby et al., 1993; Bremmer et al., 1997). Importantly, the direct comparison between both species revealed that saccade-related activity decreased while SPEMrelated activity increased from posterior to anterior/lateral in human PPC and from lateral/dorsal to ventral in monkey PPC. Thus, both human and monkey PPC exhibit a similar gradient organization in the representation of saccades and SPEMs.

Second, we used an fMR-A paradigm to probe motion selectivity in human PPC and visual cortex. The stimulus set consisted of random dots moving coherently to produce optic flow patterns including planar, circular, and radial motion (Duffy, 1998). In visual cortex, we found motion-selective responses in a distributed network of visual areas including V1, V2, V3, V4, V3A, and $\mathrm{MT}+$ similar to results in anesthetized monkeys obtained in an fMR-A paradigm (Tolias et al., 2001) and in confirmation of numerous physiology studies (Albright, 1984; Burkhalter and Van Essen, 1986; Gaska et al., 1988; Hawken et al., 1988; Schiller, 1996; Tolias et al., 2005).

Our results showed that all topographically organized areas in PPC exhibited motion-selective responses. Furthermore, IPS1IPS3 showed stronger adaptation effects induced by radial compared with planar and circular optic flow. These results are in agreement with monkey PPC, in which areas at the apex of the dorsal stream contain neurons that respond selectively to radial motion (Steinmetz et al., 1987; Schaafsma and Duysens, 1996; Siegel and Read, 1997; Bremmer et al., 2002; Schlack et al., 2002). 
For example, in area 7a, the majority of neurons preferred radial over planar and circular motion (Merchant et al., 2001). Thus, visual motion appears to be similarly represented in human and monkey PPC.

Our study contributes to a growing body of recent work that has related functional characteristics to underlying topography in human PPC. Converging evidence from previous fMRI studies together with the present results indicate that IPS1-IPS2 and SPL1 in humans exhibit similar response properties compared with LIP and area $7 \mathrm{a}$ in nonhuman primates. Physiology studies in monkeys have shown that both LIP and area $7 \mathrm{a}$, which are located adjacent to visual cortex, occupy the highest position within the visual hierarchy of the dorsal processing stream (Felleman and Van Essen, 1991). Likewise, IPS1-IPS2 and SPL1 are located just anterior to visual cortex in the posterior IPS. These areas as well as macaque LIP and area 7 a have also been shown to be involved in spatial attention and working memory (Gnadt and Andersen, 1988; Colby et al., 1996; Constantinidis and Steinmetz, 1996, 2001; Schluppeck et al., 2005; Silver et al., 2005). IPS1-IPS2 and SPL1 showed a preferred representation of saccades relative to SPEMs, similar compared with neurons in LIP and area 7a (Andersen et al., 1990; Barash et al., 1991; Colby et al., 1996; Bremmer et al., 1997). Evidence for functional homology between IPS1-IPS2 and LIP comes also from neuroimaging findings that IPS1 and IPS2 exhibit both saccade- and reach-related activity (Hagler et al., 2007; Levy et al., 2007). Both effectorspecific responses have been found in LIP neurons at the singlecell level (Snyder et al., 1997). Importantly, LIP neurons exhibit shape-selective responses (Sereno and Maunsell, 1998). So do IPS1 and IPS2, as recently demonstrated using fMR-A paradigms (Konen and Kastner, 2008). In contrast, no object selectivity has been found in SPL1 (our unpublished observation) nor has it been reported for area 7a neurons. Furthermore, SPL1 and 7a are both located adjacent to the IPS in BA7, which resides in the inferior parietal lobule in nonhuman primates and in the superior parietal lobule in humans because of interspecies differences in PPC architecture originally noted by Brodmann (1909). Finally, the present results showed a pattern of motion-selective responses in SPL1 that is similar to those found in area 7a neurons (Merchant et al., 2001). Together, human IPS1-IPS2 and SPL1 exhibit a number of similar functional properties compared with monkey LIP and 7a. It is important to note that, despite strikingly similar characteristics between human and monkey PPC, there are also discrepancies in the functional profiles of these areas across species. For example, both IPS1 and IPS2 exhibit motionselective responses, whereas neurons in LIP respond to the abrupt onsets of motion stimuli (as well as to onsets of other stimuli) but do not show motion selectivity (Kusunoki et al., 2000).

There is also converging evidence from this and a previous fMRI study that IPS5 may be equivalent to macaque VIP (Sereno and Huang, 2006). In the present study, the preference in responses to SPEMs in anterior/lateral IPS5 is in agreement with the functional characteristics of VIP neurons (Schlack et al., 2003). We also found that IPS5 responded selectively to optic flow patterns, which has been shown in macaque VIP (Schaafsma et al., 1997). The majority of VIP neurons are bimodal and respond both to tactile and visual stimulation (Colby et al., 1993; Duhamel et al., 1998). Critically, the tactile and visual receptive fields often demonstrate coaligned directional selectivity, suggesting that area VIP may serve a broader sensory-motor function for defensive behavior (Cooke et al., 2003). These characteristics are in agreement with coaligned representations of tactile and visual space in a region located around the superior part of the postcentral sulcus (Sereno and Huang, 2006). The multisensory representation of near extrapersonal space led to the conclusion that this area is the putative equivalent to monkey VIP. We suggest the "human parietal face area" and our IPS5 to be identical areas based on the anatomical location between the IPS and postcentral sulcus, the Talairach coordinates, and the functional topography. Thus, independent studies probing different functional characteristics suggest that IPS5 may be the putative human equivalent to monkey VIP, which is similarly located at the borderline between the visual and the somatosensory system. IPS5, however, did not exhibit greater selectivity for radial optic flow than for other flow patterns, which is in contrast to the response properties of VIP neurons (Schaafsma et al., 1997).

Together, human IPS1-IPS2, SPL1, and IPS5 exhibit similar functional characteristics compared with monkey LIP, 7a, and VIP. In contrast, functional homologies for human IPS3 and IPS4 remain puzzling. Because LIP, 7a, and VIP are adjacent areas in macaque IPS, it is interesting to note that IPS3 and IPS4 break the anatomical proximity between IPS1, IPS2, and IPS5. Thus, it is possible that IPS3 and IPS4 are human-specific areas evolving from the disproportional enlargement of PPC and resulting in a wider distribution of functions in human compared with monkey PPC (Brodmann, 1909; Van Essen et al., 2001). An alternative possibility is that IPS3 and IPS4 are functionally analogous to the medial intraparietal area and/or the caudal intraparietal area in monkeys. More studies are needed to pursue these alternative hypotheses further.

\section{References}

Albright TD (1984) Direction and orientation selectivity of neurons in visual area MT of the macaque. J Neurophysiol 52:1106-1130.

Andersen RA (1997) Multimodal integration for the representation of space in the posterior parietal cortex. Philos Trans R Soc Lond B Biol Sci 352:1421-1428.

Andersen RA, Bracewell RM, Barash S, Gnadt JW, Fogassi L (1990) Eye position effects on visual, memory, and saccade-related activity in areas LIP and 7a of macaque. J Neurosci 10:1176-1196.

Bandettini PA, Jesmanowicz A, Wong EC, Hyde JS (1993) Processing strategies for time-course data sets in functional MRI of the human brain. Magn Reson Med 30:161-173.

Barash S, Bracewell RM, Fogassi L, Gnadt JW, Andersen RA (1991) Saccaderelated activity in the lateral intraparietal area. I. Temporal properties; comparison with area 7a. J Neurophysiol 66:1095-1108.

Ben Hamed S, Duhamel JR, Bremmer F, Graf W (2001) Representation of the visual field in the lateral intraparietal area of macaque monkeys: a quantitative receptive field analysis. Exp Brain Res 140:127-144.

Berman RA, Colby CL, Genovese CR, Voyvodic JT, Luna B, Thulborn KR, Sweeney JA (1999) Cortical networks subserving pursuit and saccadic eye movements in humans: an FMRI study. Hum Brain Mapp 8:209-225.

Blatt GJ, Andersen RA, Stoner GR (1990) Visual receptive field organization and cortico-cortical connections of the lateral intraparietal area (area LIP) in the macaque. J Comp Neurol 299:421-445.

Brainard DH (1997) The psychophysics toolbox. Spat Vis 10:433-436.

Bremmer F, Distler C, Hoffmann KP (1997) Eye position effects in monkey cortex. II. Pursuit- and fixation-related activity in posterior parietal areas LIP and 7A. J Neurophysiol 77:962-977.

Bremmer F, Schlack A, Shah NJ, Zafiris O, Kubischik M, Hoffmann K, Zilles K, Fink GR (2001) Polymodal motion processing in posterior parietal and premotor cortex: a human fMRI study strongly implies equivalencies between humans and monkeys. Neuron 29:287-296.

Bremmer F, Klam F, Duhamel JR, Ben Hamed S, Graf W (2002) Visualvestibular interactive responses in the macaque ventral intraparietal area (VIP). Eur J Neurosci 16:1569-1586.

Brewer AA, Press WA, Logothetis NK, Wandell BA (2002) Visual areas in macaque cortex measured using functional magnetic resonance imaging. J Neurosci 22:10416-10426.

Brodmann K (1909) Vergleichende Lokalisationslehre der Grosshirnrinde 
in ihren Prinzipien dargestellt aufgrund des Zellenbaues. Leipzig, Germany: Barth.

Brown MR, DeSouza JF, Goltz HC, Ford K, Menon RS, Goodale MA, Everling S (2004) Comparison of memory- and visually guided saccades using event-related fMRI. J Neurophysiol 91:873-889.

Bruce CJ, Goldberg ME, Bushnell MC, Stanton GB (1985) Primate frontal eye fields. II. Physiological and anatomical correlates of electrically evoked eye movements. J Neurophysiol 54:714-734.

Burkhalter A, Van Essen DC (1986) Processing of color, form and disparity information in visual areas $\mathrm{VP}$ and $\mathrm{V} 2$ of ventral extrastriate cortex in the macaque monkey. J Neurosci 6:2327-2351.

Colby CL, Duhamel JR (1996) Spatial representations for action in parietal cortex. Brain Res Cogn Brain Res 5:105-115.

Colby CL, Goldberg ME (1999) Space and attention in parietal cortex. Annu Rev Neurosci 22:319-349.

Colby CL, Duhamel JR, Goldberg ME (1993) Ventral intraparietal area of the macaque: anatomic location and visual response properties. J Neurophysiol 69:902-914.

Colby CL, Duhamel JR, Goldberg ME (1996) Visual, presaccadic, and cognitive activation of single neurons in monkey lateral intraparietal area. J Neurophysiol 76:2841-2852.

Constantinidis C, Steinmetz MA (1996) Neuronal activity in posterior parietal area 7a during the delay periods of a spatial memory task. J Neurophysiol 76:1352-1355.

Constantinidis C, Steinmetz MA (2001) Neuronal responses in area 7a to multiple stimulus displays: II. Responses are suppressed at the cued location. Cereb Cortex 11:592-597.

Cooke DF, Taylor CS, Moore T, Graziano MS (2003) Complex movements evoked by microstimulation of the ventral intraparietal area. Proc Natl Acad Sci U S A 100:6163-6168.

Cox RW, Jesmanowicz A (1999) Real-time 3D image registration for functional MRI. Magn Reson Med 42:1014-1018.

Duffy CJ (1998) MST neurons respond to optic flow and translational movement. J Neurophysiol 80:1816-1827.

Duffy CJ, Wurtz RH (1991) Sensitivity of MST neurons to optic flow stimuli. I. A continuum of response selectivity to large-field stimuli. J Neurophysiol 65:1329-1345.

Duhamel JR, Colby CL, Goldberg ME (1998) Ventral intraparietal area of the macaque: congruent visual and somatic response properties. J Neurophysiol 79:126-136.

Efron B, Tibshirani R (1991) Statistical data analysis in the computer age. Science 253:390-395.

Engel SA, Glover GH, Wandell BA (1997) Retinotopic organization in human visual cortex and the spatial precision of functional MRI. Cereb Cortex 7:181-192.

Felleman DJ, Van Essen DC (1991) Distributed hierarchical processing in the primate cerebral cortex. Cereb Cortex 1:1-47.

Friston KJ, Frith CD, Turner R, Frackowiak RS (1995) Characterizing evoked hemodynamics with fMRI. Neuroimage 2:157-165.

Gaska JP, Jacobson LD, Pollen DA (1988) Spatial and temporal frequency selectivity of neurons in visual cortical area $\mathrm{V} 3 \mathrm{~A}$ of the macaque monkey. Vision Res 28:1179-1191.

Gitelman DR (2002) ILAB: a program for postexperimental eye movement analysis. Behav Res Methods Instrum Comput 34:605-612.

Gnadt JW, Andersen RA (1988) Memory related motor planning activity in posterior parietal cortex of macaque. Exp Brain Res 70:216-220.

Goodale MA, Milner AD (1992) Separate visual pathways for perception and action. Trends Neurosci 15:20-25.

Gottlieb JP, Bruce CJ, MacAvoy MG (1993) Smooth eye movements elicited by microstimulation in the primate frontal eye field. J Neurophysiol 69:786-799.

Graziano MS, Andersen RA, Snowden RJ (1994) Tuning of MST neurons to spiral motions. J Neurosci 14:54-67.

Grefkes C, Fink GR (2005) The functional organization of the intraparietal sulcus in humans and monkeys. J Anat 207:3-17.

Grefkes C, Weiss PH, Zilles K, Fink GR (2002) Crossmodal processing of object features in human anterior intraparietal cortex: an fMRI study implies equivalencies between humans and monkeys. Neuron 35:173-184.

Grill-Spector K, Kushnir T, Edelman S, Avidan G, Itzchak Y, Malach R (1999) Differential processing of objects under various viewing conditions in the human lateral occipital complex. Neuron 24:187-203.
Hadjikhani N, Liu AK, Dale AM, Cavanagh P, Tootell RB (1998) Retinotopy and color sensitivity in human visual cortical area V8. Nat Neurosci 1:235-241.

Hagler DJ Jr, Riecke L, Sereno MI (2007) Parietal and superior frontal visuospatial maps activated by pointing and saccades. Neuroimage 35:1562-1577.

Hawken MJ, Parker AJ, Lund JS (1988) Laminar organization and contrast sensitivity of direction-selective cells in the striate cortex of the Old World monkey. J Neurosci 8:3541-3548.

Huerta MF, Krubitzer LA, Kaas JH (1986) Frontal eye field as defined by intracortical microstimulation in squirrel monkeys, owl monkeys, and macaque monkeys: I. Subcortical connections. J Comp Neurol 253:415-439.

Huk AC, Dougherty RF, Heeger DJ (2002) Retinotopy and functional subdivision of human areas MT and MST. J Neurosci 22:7195-7205.

Kastner S, De Weerd P, Desimone R, Ungerleider LG (1998) Mechanisms of directed attention in the human extrastriate cortex as revealed by functional MRI. Science 282:108-111.

Kastner S, DeSimone K, Konen CS, Szczepanski SM, Weiner KS, Schneider KA (2007) Topographic maps in human frontal cortex revealed in memory-guided saccade and spatial working-memory tasks. J Neurophysiol 97:3494-3507.

Konen CS, Kastner S (2008) Two hierarchically organized neural systems for object information in human visual cortex. Nat Neurosci 11:224-231.

Konen CS, Kleiser R, Wittsack HJ, Bremmer F, Seitz RJ (2004) The encoding of saccadic eye movements within human posterior parietal cortex. Neuroimage 22:304-314.

Konen CS, Kleiser R, Seitz RJ, Bremmer F (2005) An fMRI study of optokinetic nystagmus and smooth-pursuit eye movements in humans. Exp Brain Res 165:203-216.

Kusunoki M, Gottlieb J, Goldberg ME (2000) The lateral intraparietal area as a salience map: the representation of abrupt onset, stimulus motion, and task relevance. Vision Res 40:1459-1468.

Lagae L, Maes H, Raiguel S, Xiao DK, Orban GA (1994) Responses of macaque STS neurons to optic flow components: a comparison of areas MT and MST. J Neurophysiol 71:1597-1626.

Levy I, Schluppeck D, Heeger DJ, Glimcher PW (2007) Specificity of human cortical areas for reaches and saccades. J Neurosci 27:4687-4696.

Luna B, Thulborn KR, Strojwas MH, McCurtain BJ, Berman RA, Genovese CR, Sweeney JA (1998) Dorsal cortical regions subserving visually guided saccades in humans: an fMRI study. Cereb Cortex 8:40-47.

Maunsell JH, Van Essen DC (1983) Functional properties of neurons in middle temporal visual area of the macaque monkey. II. Binocular interactions and sensitivity to binocular disparity. J Neurophysiol 49:1148-1167.

Merchant H, Battaglia-Mayer A, Georgopoulos AP (2001) Effects of optic flow in motor cortex and area 7a. J Neurophysiol 86:1937-1954.

Pelli DG (1997) The VideoToolbox software for visual psychophysics: transforming numbers into movies. Spat Vis 10:437-442.

Petit L, Clark VP, Ingeholm J, Haxby JV (1997) Dissociation of saccaderelated and pursuit-related activation in human frontal eye fields as revealed by fMRI. J Neurophysiol 77:3386-3390.

Pierrot-Deseilligny C, Rivaud S, Gaymard B, Müri R, Vermersch AI (1995) Cortical control of saccades. Ann Neurol 37:557-567.

Press WA, Brewer AA, Dougherty RF, Wade AR, Wandell BA (2001) Visual areas and spatial summation in human visual cortex. Vision Res 41:1321-1332.

Robinson DA, Fuchs AF (1969) Eye movements evoked by stimulation of frontal eye fields. J Neurophysiol 32:637-648.

Rosano C, Krisky CM, Welling JS, Eddy WF, Luna B, Thulborn KR, Sweeney JA (2002) Pursuit and saccadic eye movement subregions in human frontal eye field: a high-resolution fMRI investigation. Cereb Cortex 12:107-115.

Saito H, Yukie M, Tanaka K, Hikosaka K, Fukada Y, Iwai E (1986) Integration of direction signals of image motion in the superior temporal sulcus of the macaque monkey. J Neurosci 6:145-157.

Schaafsma SJ, Duysens J (1996) Neurons in the ventral intraparietal area of awake macaque monkey closely resemble neurons in the dorsal part of the medial superior temporal area in their responses to optic flow patterns. J Neurophysiol 76:4056-4068.

Schaafsma SJ, Duysens J, Gielen CC (1997) Responses in ventral intraparietal area of awake macaque monkey to optic flow patterns corresponding 
to rotation of planes in depth can be explained by translation and expansion effects. Vis Neurosci 14:633-646.

Schiller PH (1996) On the specificity of neurons and visual areas. Behav Brain Res 76:21-35.

Schlack A, Hoffmann KP, Bremmer F (2002) Interaction of linear vestibular and visual stimulation in the macaque ventral intraparietal area (VIP). Eur J Neurosci 16:1877-1886.

Schlack A, Hoffmann KP, Bremmer F (2003) Selectivity of macaque ventral intraparietal area (area VIP) for smooth pursuit eye movements. J Physiol 551:551-561.

Schluppeck D, Glimcher P, Heeger DJ (2005) Topographic organization for delayed saccades in human posterior parietal cortex. J Neurophysiol 94:1372-1384.

Schluppeck D, Curtis CE, Glimcher PW, Heeger DJ (2006) Sustained activity in topographic areas of human posterior parietal cortex during memory-guided saccades. J Neurosci 26:5098-5108.

Schneider KA, Richter MC, Kastner S (2004) Retinotopic organization and functional subdivisions of the human lateral geniculate nucleus: a highresolution functional magnetic resonance imaging study. J Neurosci 24:8975-8985.

Sereno AB, Maunsell JH (1998) Shape selectivity in primate lateral intraparietal cortex. Nature 395:500-503.

Sereno MI, Huang RS (2006) A human parietal face area contains aligned head-centered visual and tactile maps. Nat Neurosci 9:1337-1343.

Sereno MI, Dale AM, Reppas JB, Kwong KK, Belliveau JW, Brady TJ, Rosen BR, Tootell RB (1995) Borders of multiple visual areas in humans revealed by functional magnetic resonance imaging. Science 268:889-893.

Sereno MI, Pitzalis S, Martinez A (2001) Mapping of contralateral space in retinotopic coordinates by a parietal cortical area in humans. Science 294:1350-1354.

Siegel RM, Read HL (1997) Analysis of optic flow in the monkey parietal area 7a. Cereb Cortex 7:327-346.

Siegel RM, Raffi M, Phinney RE, Turner JA, Jandó G (2003) Functional architecture of eye position gain fields in visual association cortex of behaving monkey. J Neurophysiol 90:1279-1294.

Silver MA, Ress D, Heeger DJ (2005) Topographic maps of visual spatial attention in human parietal cortex. J Neurophysiol 94:1358-1371.

Snyder LH, Batista AP, Andersen RA (1997) Coding of intention in the posterior parietal cortex. Nature 386:167-170.
Stanton GB, Goldberg ME, Bruce CJ (1988) Frontal eye field efferents in the macaque monkey: I. Subcortical pathways and topography of striatal and thalamic terminal fields. J Comp Neurol 271:473-492.

Steinmetz MA, Motter BC, Duffy CJ, Mountcastle VB (1987) Functional properties of parietal visual neurons: radial organization of directionalities within the visual field. J Neurosci 7:177-191.

Swisher JD, Halko MA, Merabet LB, McMains SA, Somers DC (2007) Visual topography of human intraparietal sulcus. J Neurosci 27:5326-5337.

Talairach J, Tournoux P (1988) Co-planar stereotaxic atlas of the human brain. Stuttgart, Germany: Thieme.

Thier P, Andersen RA (1998) Electrical microstimulation distinguishes distinct saccade-related areas in the posterior parietal cortex. J Neurophysiol 80:1713-1735.

Tolias AS, Smirnakis SM, Augath MA, Trinath T, Logothetis NK (2001) Motion processing in the macaque: revisited with functional magnetic resonance imaging. J Neurosci 21:8594-8601.

Tolias AS, Keliris GA, Smirnakis SM, Logothetis NK (2005) Neurons in macaque area $\mathrm{V} 4$ acquire directional tuning after adaptation to motion stimuli. Nat Neurosci 8:591-593.

Tootell RB, Mendola JD, Hadjikhani NK, Ledden PJ, Liu AK, Reppas JB, Sereno MI, Dale AM (1997) Functional analysis of V3A and related areas in human visual cortex. J Neurosci 17:7060-7078.

Tootell RB, Mendola JD, Hadjikhani NK, Liu AK, Dale AM (1998) The representation of the ipsilateral visual field in human cerebral cortex. Proc Natl Acad Sci U S A 95:818-824.

Ungerleider LG, Mishkin M (1982) Two cortical visual systems. In: Analysis of visual behavior (Ingle DJ, Goodale MA, Mansfield RJW, eds), pp 549586. Cambridge, MA: MIT.

Van Essen DC, Felleman DJ, DeYoe EA, Olavarria J, Knierim J (1990) Modular and hierarchical organization of extrastriate visual cortex in the macaque monkey. Cold Spring Harb Symp Quant Biol 55:679-696.

Van Essen DC, Lewis JW, Drury HA, Hadjikhani N, Tootell RB, Bakircioglu M, Miller MI (2001) Mapping visual cortex in monkeys and humans using surface-based atlases. Vision Res 41:1359-1378.

Wade AR, Brewer AA, Rieger JW, Wandell BA (2002) Functional measurements of human ventral occipital cortex: retinotopy and colour. Philos Trans R Soc Lond B Biol Sci 357:963-973. 